\title{
Roaming Under the Microscope: Trajectory Study of Formaldehyde
}

\section{Dissociation}

\author{
Paul L. Houston, ${ }^{\dagger}$ Riccardo Conte,,,$+ \uparrow$ and Joel M. Bowman ${ }^{*, \ddagger}$ \\ $\dagger$ School of Chemistry and Biochemistry Georgia Institute of Technology Atlanta, Georgia \\ 30332, USA., Department of Chemistry and Chemical Biology, Cornell University, Baker \\ Laboratory, Ithaca, NY 14852, USA. \\ $\ddagger$ Department of Chemistry and Cherry L. Emerson Center for Scientific Computation, \\ Emory University Atlanta, Georgia 30322, USA. \\ ๑ Dipartimento di Chimica, Università degli Studi di Milano, 20133 Milano, Italy. email: \\ riccardo.conte1@unimi.it
}

E-mail: jmbowma@emory.edu,1-404-727-6592 


\begin{abstract}
The photodissociation of formaldehyde has been studied using quasi-classical trajectories to investigate "roaming," or events involving trajectories that proceed far from the minimum energy pathway. Statistical analysis of trajectories performed over a range of nine excitation energies from 34500 to $41010 \mathrm{~cm}^{-1}$ (including zpe) provides characterization of the roaming phenomenon and insight into the mechanism. The trajectories are described as projections onto three coordinates: the distance from the CO center of mass to the furthest $\mathrm{H}$ atom and the azimuthal and polar coordinates of that $\mathrm{H}$ atom with respect to the $\mathrm{CO}$ axis. The trajectories are used to construct a "minimum energy" potential energy surface showing the potential for any binary combination of these three coordinates that is at a minimum energy with respect to values of the other coordinates encountered during the trajectories. We also construct flux diagrams for roaming, transition-state and radical pathways, as well as "reaction configuration" plots that show the distribution of reaction geometries for roaming and transition-state pathways. These constructs allow characterization of roaming in formaldehyde as, principally, internal rotation of the roaming $\mathrm{H}$ atom around the $\mathrm{CO}$ axis at a slowly varying and elongated distance from the CO center of mass. The rotation is nearly uniform, and is sometimes accompanied by rotation in the polar coordinate. The "roaming" state of formaldehyde can be treated as a separate kinetic entity, much as one might treat an isomer. Rate constants for the formation of and reaction from this roaming state are derived from the trajectory data as a function of excitation energy.
\end{abstract}

Keywords: dissociation dynamics, transition-state theory, quasi-classical trajectories, roaming, kinetics. 


\section{Introduction}

Transition-state theory has been a bedrock method for calculating the rate constants of chemical processes, ${ }^{1-3}$ yet, at least in its most common form, it is based on the notion that molecular motions predominantly follow a "minimum energy path." There is increasing evidence that highly-excited molecules often bypass this minimum energy path and react in unexpected ways from unexpected geometries. An early, confirmed example of this behavior, often called "roaming," was stimulated by and speculated about in experimental work on formaldehyde dissociation in the research groups of Moore,${ }^{4-7}$ and then confirmed by the research groups of Suits, ${ }^{8-13}$ and Bowman. ${ }^{8,10-16}$ Reviews of this research ${ }^{17-20}$ and further studies of formaldehyde dissociation and the reverse $\mathrm{H}+\mathrm{HCO}$ reaction ensued. ${ }^{21-25}$ Similar roaming behavior has subsequently been found in other unimolecular dissociations, including those of acetaldehyde, ${ }^{26-33}$, larger aldehydes, ${ }^{34}$ methyl formate,${ }^{35-39}$ acetone, ${ }^{40}$ alkanes, ${ }^{41} \mathrm{NO}_{3},{ }^{23,42,43}$ nitromethane, ${ }^{44-46}$ methyl nitrite, ${ }^{45}$ carbon dioxide,${ }^{47}$ and Criegee intermediates. ${ }^{48}$ Roaming in bimolecular reactions has also been observed and discussed. ${ }^{49-51}$ Several reviews of this active area of research have recently appeared. ${ }^{52-54}$ Consequently, it is now well established that many systems react via trajectories that deviate strongly from the minimum energy path. The consequence of this behavior for transition-state theory is still incompletely understood.

In this paper we focus on the detailed mechanism of roaming in the dissociation of formaldehyde. Previous papers presented examples of roaming trajectories and showed clearly the large amplitude motion associated with it in regions of high potential and thus low relative kinetic energy of the roaming $\mathrm{H}$ atom and the $\mathrm{HCO}$ fragment. Here, many roaming trajectories are examined and processed to develop a more complete and comprehensive characterization of roaming. Nine excitation energies have been studied, from 34500 to $41010 \mathrm{~cm}^{-1}$ above the global minimum of the formaldehyde well. From the trajectories, we find the "minimum energy" potential, showing clearly a plateau region in potential that is traversed by the roaming $\mathrm{H}$ atom. At each energy, we develop flux maps for the roaming 
and TS pathways to $\mathrm{H}_{2}+\mathrm{CO}$ and for the reaction to $\mathrm{H}+\mathrm{HCO}$. We also construct reaction configuration plots, showing the distribution of reaction geometries for roaming and TS trajectories. Average quantities for many measurable properties of the trajectories are tabulated as a function of excitation energy.

From these observations, we find that roaming involves, principally, an internal rotation in which the $\mathrm{H}$ atom rotates around the $\mathrm{CO}$ bond at a slowly-varying and elongated distance from the $\mathrm{CO}$ center of mass. This azimuthal rotation is often accompanied by a rotation in the polar angle, but the azimuthal rotation is most prominent. Reaction proceeds from a stretched $\mathrm{C}-\mathrm{H}-\mathrm{H}$ geometry that leads to substantial $\mathrm{H}_{2}$ vibrational energy. There is a strong correlation between the internal energy of the $\mathrm{H}_{2}$ and that of the $\mathrm{CO}$, such that roaming trajectories have much higher $\mathrm{H}_{2}$ internal energy and lower $\mathrm{CO}$ internal energy. We find further that the roaming state can be treated as a separate kinetic entity, much as one would treat an isomer. Rate constants for formation of and reaction from this roaming state are calculated at each energy based on the results of the trajectory calculations. This finding suggests that transition state theories should still be effective in calculating rate constants, provided that separate regions of the PES are treated as separate kinetic entities.

Table 1 lists acronyms used in the text.

Table 1: Acronyms used in the text

\begin{tabular}{|c|c|}
\hline Acronym & Meaning \\
\hline \hline COM & center of mass \\
\hline FWHM & full width at half maximum \\
\hline KE & kinetic energy \\
\hline PE & potential energy \\
\hline PES & potential energy surface \\
\hline TS & transition state \\
\hline zpe & zero point energy \\
\hline
\end{tabular}




\section{Potential Energy Surface and Trajectory Methods}

The global potential energy surface (PES) adopted to run trajectories has been described previously. ${ }^{14}$ In short, we recall that the PES was obtained upon sampling of 80000 CCSD(T)/augcc-PVTZ ab initio energies covering $\mathrm{H}_{2} \mathrm{CO}$ global minimum and isomer geometries as well as the molecular $\mathrm{H}_{2}+\mathrm{CO}$ channel. For the radical dissociation channel $\mathrm{H}+\mathrm{HCO}$ and for other regions of the global surface which could not be characterized by a single-reference method, more than 50000 MRCI/aug-cc-PVTZ energies have been collected. The global PES is constituted by a total of six local fits, smoothly joined by switching functions.

The trajectory methods are similar to those used previously. ${ }^{55-62}$ We started quasiclassical trajectories from the global minimum geometry of $\mathrm{H}_{2} \mathrm{CO}$. The total internal excitation, including zpe, was sampled at nine different values (i.e. 34500, 35000, 35500, $36000,36223,37688,38688,38814$, and $41010 \mathrm{~cm}^{-1}$ ). To get the right amount of excitation, we initially assigned random velocities to all four atoms. We then set the total angular momentum J to 0 by subtracting the angular velocity contribution, and finally rescaled all velocities to the chosen value of the now purely vibrational excitation energy. Since we aim at investigating "roaming" events, which involve large amplitude motions, a large-distance exit condition for trajectories has been employed, i.e. a trajectory is stopped only when any two atoms are at least 200 a.u. away. The total energy was typically conserved along trajectories with an accuracy of 1 part in $10^{3}$ or better.

The calculations produced printout "frames" of the trajectory every 6.25 fs (125 steps at

$0.05 \mathrm{fs} / \mathrm{step})$ providing the positions and velocities of the four atoms. Analysis of the trajectories was performed by first evaluating the last frame to determine whether the reaction products were $\mathrm{H}_{2}+\mathrm{CO}$ or $\mathrm{H}+\mathrm{HCO}$ and then determining properties of the reaction from either the last frame or from an average of frames near the one in which reaction was determined to have occurred (see below for definition of this "reaction frame"). Typical properties in the case of reaction to $\mathrm{H}_{2}+\mathrm{CO}$ were the vibrational and rotational energies of each of the products, the angular momentum of each of the products, the relative velocity and trans- 
lational energy of the products, their orbital angular momentum and its associated energy, the final impact parameter for the departing fragments, the $\mathbf{v}-\mathbf{J}$ correlation for each of the products, the alignment of their rotational vectors relative to the transition dipole as well as relative to one another, and the anisotropy of dissociation. Also tabulated were the average kinetic energy, the average number of $\mathrm{CH}$ bonds, the average $\mathrm{H}-\mathrm{H}$ bond length, and the average values of the coordinates $r_{H}, \theta, \phi$ (defined below), where the average was taken over the 50 frames prior to the reaction frame. In the case of reaction to $\mathrm{H}+\mathrm{HCO}$, we tabulated the angular momentum of the $\mathrm{HCO}$, the relative velocity and translational energy for the $\mathrm{H}$ and $\mathrm{HCO}$, the orbital energy, and the vibrational and rotational energy of the HCO. For each group of trajectories at a total excitation energy, distributions of these properties were constructed, and an average was determined from each distribution. From a distribution of the dissociation times, a plot was made of the log of the unreacted trajectories as a function of time, and this plot was used to determine the total reaction rate at each excitation energy. Finally, for those trajectories that were assigned as "roaming", a distribution of the "roaming time" was determined as the time prior to the reaction frame during which the kinetic energy was less than $25 \%$ of the total excitation energy. An average roaming time was calculated from the distribution.

Trajectories are projected onto pairs of coordinates in a three-dimensional system that includes $r_{H}$, the distance from the $\mathrm{CO}$ center of mass to the $\mathrm{H}$ atom that is most distant; $\theta$, the polar angle between a line along $r_{H}$ and the CO bond, with $\theta=0$ being defined as having the most distant $\mathrm{H}$ atom nearer the carbon; and $\phi$, the azimuthal angle between the two planes containing the $\mathrm{CO}$ bond and either $\mathrm{H}$ atom, where $\phi=\pi$ corresponds to having all atoms in the same plane with the $\mathrm{H}$ atoms on opposite sides of the $\mathrm{CO}$ bond. The ranges for $\theta$ and $\phi$ are both from 0 to $\pi$. There are $3 N-6$ internal coordinates for formaldehyde, so that the values of three coordinates are unspecified. If the trajectory is being projected onto, for example, the $r_{H}, \phi$ plane, any trajectory values of $\theta$ and of the three unspecified coordinates will be projected to the same point on the $r_{H}, \phi$ plane. 
A well-known problem of quasi-classical trajectory studies is referred to as "zero-point energy leakage," the "flow" of energy from the quantum-mechanically required zero-point vibration into other degrees of freedom. ${ }^{63}$ When the excitation energy far exceeds threshold values, this leakage is not normally a problem, but when, as in the current case of roaming, the energies are close to threshold, zero-point leakage can influence the dynamics, particularly the branching ratios to different product channels. Indeed, nearly $75 \%$ of the trajectories at the energies studied here had zero-point energy violations, that is, values of the final vibrational energy for a species that were below that required by quantum mechanics. Some further statistics illustrate the situation: as the excitation energy increased from the lowest to the highest ones studied, i) the fraction of $\mathrm{H}_{2}+\mathrm{CO}$ reactions that violated zpe decreased from 0.70 to 0.41 and the fraction of these due to $\mathrm{CO}$ zpe violation decreased from 0.87 to 0.74., and ii) the fraction of $\mathrm{H}+\mathrm{HCO}$ reactions that violated zpe decreased from 1.00 to 0.33. For those trajectories that violated zpe requirements, the average fraction of the zpe in $\mathrm{CO}$ was about 0.46 ; in $\mathrm{H}_{2}$ it was about 0.45 , and in $\mathrm{HCO}$ it ranged from 0.30 to 0.77 with increasing energy. We thus paid careful attention to the leakage issue by calculating dynamical properties in two limits: a) exclusion of those trajectories that had zero-point violations for one or more product, and b) inclusion of all trajectories that were otherwise acceptable. We found that many of the average properties we characterized were nearly identical for the two groups; that is, the average values were within about $10 \%$ of each other, which is about the uncertainty in either value. Thus, the numbers reported below should be regarded as accurate to $\pm 10 \%$. In the case of the few properties for which the average values varied by more than $10 \%$ between the groups, we report in the tables below those values from group a). Values taken from this group are indicated by bold-faced type. The properties whose values are determined from group a) include the average vibrational energies of the $\mathrm{CO}$ and $\mathrm{H}_{2}$ and the branching ratios between the $\mathrm{H}_{2}+\mathrm{CO}$ and $\mathrm{H}+\mathrm{HCO}$ channels.

The de Broglie wavelength for a roaming $\mathrm{H}$ atom can be estimated from figures to be introduced below. It is about $0.8 \AA$, considerably less than the typical distance of the 
roaming atom from the $\mathrm{HCO}$ molecule, $>3 \AA$. Thus, for most cases, it is reasonable to treat the $\mathrm{H}$ atom classically. The possibility of tunneling depends on the potential. We have not included corrections for this effect. 


\section{Results}

\section{Overview}

A general overview of the trajectory results is shown in Table 2, which compares outcomes as a function of excitation energy. All trajectories were run until reaction, and the net number of reactions at each energy ranged from about 4000 to 4600 . The percentage of molecular products decreases from $100 \%$ at the lowest energy $\left(34500 \mathrm{~cm}^{-1}\right)$ to about $42 \%$ at the highest energy $\left(41010 \mathrm{~cm}^{-1}\right)$, with the remaining percentage going to radical products. The fraction of trajectories that exhibit roaming just prior to reaction to give molecular products increases from about $2.6 \%$ to about $14 \%$ over this range of energies. Roaming with subsequent reaction to molecular products via the TS pathway was also observed at about $1 \%$ of the total reactions producing $\mathrm{H}_{2}+\mathrm{CO}$. It was more difficult to quantify reactions that exhibited roaming before dissociation to radical products, but this pathway was observed at percentage levels that ranged from $0 \%$ to $10 \%$.

Table 2: General Properties of Formaldehyde Dissociation Trajectories*

\begin{tabular}{|c|c|c|c|c|c|c|c|c|c|c|}
\hline Line & Property/Etot $\left(\mathrm{k} \mathrm{cm}^{-1}\right)$ & 34.5 & 35.0 & 35.5 & 36.0 & 36.223 & 37.688 & 38.688 & 38.814 & 41.010 \\
\hline \hline 1a & Number of Reactions group a) $\dagger$ & $\mathbf{9 8 2}$ & $\mathbf{9 3 8}$ & $\mathbf{9 4 0}$ & $\mathbf{9 8 8}$ & $\mathbf{1 0 6 0}$ & $\mathbf{2 4 6 6}$ & $\mathbf{3 0 0 3}$ & $\mathbf{3 0 0 8}$ & $\mathbf{3 4 9 1}$ \\
\hline 1b & Number of Reactions group b) $\dagger$ & 4080 & 4256 & 4423 & 4475 & 4486 & 4615 & 4627 & 4599 & 4643 \\
\hline 2 & $\mathrm{H}_{2}+\mathrm{CO}(\%)$ & $\mathbf{1 0 0 . 0}$ & $\mathbf{1 0 0 . 0}$ & $\mathbf{1 0 0 . 0}$ & $\mathbf{1 0 0 . 0}$ & $\mathbf{9 7 . 3}$ & $\mathbf{4 4 . 4}$ & $\mathbf{4 3 . 7}$ & $\mathbf{4 2 . 9}$ & $\mathbf{4 2 . 1}$ \\
\hline 3 & $\mathrm{H}+\mathrm{HCO}(\%)$ & $\mathbf{0}$ & $\mathbf{0}$ & $\mathbf{0}$ & $\mathbf{0}$ & $\mathbf{2 . 7}$ & $\mathbf{5 5 . 6}$ & $\mathbf{5 6 . 3}$ & $\mathbf{5 7 . 1}$ & $\mathbf{5 7 . 9}$ \\
\hline 4 & Roaming to $\mathrm{H}_{2}+\mathrm{CO}(\%)$ & $\mathbf{2 . 6}$ & $\mathbf{6 . 8}$ & $\mathbf{9 . 1}$ & $\mathbf{8 . 2}$ & $\mathbf{9 . 8}$ & $\mathbf{9 . 3}$ & $\mathbf{1 5 . 1}$ & $\mathbf{1 2 . 6}$ & $\mathbf{1 3 . 5}$ \\
\hline 5 & $\begin{array}{c}\text { Roaming, reentering well } \\
\text { and then TS to } \mathrm{H}_{2}+\mathrm{CO}(\%)\end{array}$ & $\mathbf{0 . 7}$ & $\mathbf{1 . 7}$ & $\mathbf{1 . 6}$ & $\mathbf{1 . 8}$ & $\mathbf{0 . 7}$ & $\mathbf{0 . 7}$ & $\mathbf{0 . 6}$ & $\mathbf{0 . 9}$ & $\mathbf{0 . 5}$ \\
\hline 6 & Roaming to H + HCO $(\%)$ & $\mathbf{0}$ & $\mathbf{0}$ & $\mathbf{0}$ & $\mathbf{0}$ & $>\mathbf{1 0 . 3}$ & $>\mathbf{2 . 6}$ & $>\mathbf{2 . 3}$ & $>\mathbf{2 . 4}$ & $>\mathbf{2 . 3}$ \\
\hline
\end{tabular}

${ }^{*} \mathrm{H}_{2}+\mathrm{CO}$ and $\mathrm{H}+\mathrm{HCO}$ percentages are of net reactions, other percentages are of relevant reactions. Boldface values are based on group a).

†See text in Section 2 for explanation of groups.

A detailed examination of the properties measured is provided in Table 3, based on averages from individual distribution plots at each energy. This table shows the results for only three energies; those for all nine energies are included in Table S1. Table S2 explains acronyms and provides brief descriptions of the properties. 
Table 3: Average values of measured trajectory properties (for which brief descriptions are given in Table S2).

\begin{tabular}{|c|c|c|c|c|}
\hline Line & Property/Energy $\left(\mathrm{k} \mathrm{cm}^{-1}\right)$ & 35.000 & 36.223 & 41.010 \\
\hline 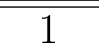 & Photon energy $\left(\mathrm{k} \mathrm{cm}^{-1}\right)$ & 29.141 & 30.364 & 35.151 \\
\hline 2 & Excitation Band & $4^{3}$ & $2^{1} 4^{3}$ & $2^{6} 4^{1}$ \\
\hline 3 & TS Fract with 2 bonds* & 0.18 & .20 & 0.24 \\
\hline 4 & Roam Fract with 2 bonds* & 0.64 & .53 & 0.38 \\
\hline 5 & TS KE Fract* & 0.43 & .42 & 0.41 \\
\hline 6 & Roam KE Fract* & 0.23 & .29 & 0.36 \\
\hline 7 & TS H-H bond length $(\AA) *$ & 2.1 & 2.1 & 2.1 \\
\hline 8 & Roam H-H bond length $(\AA)^{*}$ & 2.7 & 2.5 & 2.3 \\
\hline 9 & $\mathrm{TS} \mathrm{J}(\mathrm{CO})$ & 42.3 & 41.0 & 37.6 \\
\hline 10 & Roam J(CO) & 19.5 & 20.2 & 22.9 \\
\hline 11 & $\mathrm{TS} \mathrm{J}\left(\mathrm{H}_{2}\right)$ & 4.3 & 4.9 & 7. \\
\hline 12 & Roam J $\left(\mathrm{H}_{2}\right)$ & 8.4 & 9.9 & 11.1 \\
\hline 13 & $\mathrm{TS} L_{\text {orb }}$ & 43.6 & 42.4 & 39.2 \\
\hline 14 & Roam $L_{\text {orb }}$ & 19.7 & 22.0 & 25.4 \\
\hline 15 & $\mathrm{TS} r_{H}(\AA) *$ & 1.89 & 1.9 & 1.91 \\
\hline 16 & $\operatorname{Roam} r_{H}(\AA) *$ & 2.82 & 2.54 & 2.19 \\
\hline 17 & $\mathrm{TS} \theta^{*}$ & 0.67 & 0.67 & 0.67 \\
\hline 18 & $\operatorname{Roam} \theta^{*}$ & 0.87 & 0.78 & 0.7 \\
\hline 19 & $\mathrm{TS} \phi^{*}$ & 2.61 & 2.57 & 2.53 \\
\hline 20 & $\operatorname{Roam} \phi^{*}$ & 2.08 & 2.19 & 2.38 \\
\hline 21 & $\mathrm{TS} \beta_{0}^{0}(22)(\mathrm{CO})$ & -0.5 & -0.5 & -0.49 \\
\hline 22 & Roam $\beta_{0}^{0}(22)(\mathrm{CO})$ & -0.47 & -0.45 & -0.46 \\
\hline 23 & $\mathrm{TS} \beta_{0}^{0}(22)\left(\mathrm{H}_{2}\right)$ & -0.28 & -0.25 & -0.22 \\
\hline 24 & Roam $\beta_{0}^{0}(22)\left(\mathrm{H}_{2}\right)$ & -0.37 & -0.4 & -0.35 \\
\hline 25 & $\mathrm{TS} \mathbf{J}(\mathbf{C O}) \bullet \mathbf{J}\left(\mathbf{H}_{\mathbf{2}}\right)$ & 0.21 & 0.17 & 0.08 \\
\hline 26 & Roam $\mathbf{J}(\mathbf{C O}) \bullet . \mathbf{J}\left(\mathbf{H}_{2}\right)$ & -0.30 & -0.12 & -0.05 \\
\hline 27 & $\mathrm{TS} A_{0}^{(2)}(\mathrm{CO})$ & 0.02 & 0.03 & 0.06 \\
\hline 28 & $\operatorname{Roam} A_{0}^{(2)}(\mathrm{CO})$ & 0. & 0.04 & 0.06 \\
\hline 29 & $\mathrm{TS} A_{0}^{(2)}\left(\mathrm{H}_{2}\right)$ & 0. & -0.03 & -0.05 \\
\hline 30 & $\operatorname{Roam} A_{0}^{(2)}\left(\mathrm{H}_{2}\right)$ & -0.02 & 0. & -0.01 \\
\hline 31 & $\mathrm{TS} \beta$ & 0.006 & 0.002 & -0.065 \\
\hline 32 & $\operatorname{Roam} \beta$ & -0.011 & -0.102 & -0.136 \\
\hline 33 & $\mathrm{TS} E_{v i b}(\mathrm{CO})\left(\mathrm{cm}^{-1}\right) \dagger$ & 1970. & 2210 & 3350 \\
\hline 34 & Roam $E_{v i b}(\mathrm{CO})\left(\mathrm{cm}^{-1}\right) \dagger$ & 1670 & 2000 & 2900 \\
\hline 35 & $\mathrm{TS} E_{v i b}\left(\mathrm{H}_{2}\right)\left(\mathrm{cm}^{-1}\right) \dagger$ & 6850 & 7530 & 10200 \\
\hline 36 & Roam $E_{v i b}\left(\mathrm{H}_{2}\right)\left(\mathrm{cm}^{-1}\right) \dagger$ & 21200 & 21600 & 22800 \\
\hline 37 & TS $E_{\text {trans }}\left(\mathrm{cm}^{-1}\right)$ & 20700 & 20800 & 21200 \\
\hline 38 & Roam $E_{\text {trans }}\left(\mathrm{cm}^{-1}\right)$ & 6220 & 6520 & 8430 \\
\hline 39 & $\mathrm{TS} E_{\text {orb }}\left(\mathrm{cm}^{-1}\right)$ & 2 & 1 & 1 \\
\hline 40 & Roam $E_{\text {orb }}\left(\mathrm{cm}^{-1}\right)$ & 0 & 0 & 1 \\
\hline
\end{tabular}




\begin{tabular}{|c|c|c|c|c|}
\hline 41 & TS final impact param $(\AA)$ & 0.92 & 0.89 & 0.84 \\
\hline 42 & Roam final impact param $(\AA)$ & 0.77 & 0.86 & 0.93 \\
\hline 43 & $\mathrm{TS} r_{H}, \theta, \phi$ at rxn frame & $2.10,0.82,0.52$ & $2.20,0.79,0.55$ & $2.40,0.73,0.61$ \\
\hline 44 & Roam $r_{H}, \theta, \phi$ at rxn frame & $2.80,0.90,0.38$ & $2.90,0.91,0.41$ & $3.10 .0 .83,0.51$ \\
\hline 45 & Dissociation rate constant $\left(\mathrm{ps}^{-1}\right)$ & 0.0371 & 0.1311 & 1.464 \\
\hline 46 & Roaming time $(\mathrm{ps}) \dagger$ & $\mathbf{0 . 3 1 0}$ & $\mathbf{0 . 2 1 0}$ & $\mathbf{0 . 0 7 0}$ \\
\hline
\end{tabular}

*Average over the 50 frames preceding the reaction frame

†See text in Section 2 for explaination of bold face values, which are based on group a).

\section{Depiction of the potential energy surface and features of the trajec- tories}

The potential energy surface is based on the work of Zhang et al. ${ }^{14}$ as described above. On this fitted surface, based on a zero of energy at the global minimum of the $\mathrm{H}_{2} \mathrm{CO}$ potential energy surface, the radical products $\mathrm{H}+\mathrm{HCO}$ are at an energy of $33239 \mathrm{~cm}^{-1}$ and the transition state to $\mathrm{H}_{2}+\mathrm{CO}$ products is at $30584 \mathrm{~cm}^{-1}$ (close to the ab initio value ${ }^{64}$ of $30683 \mathrm{~cm}^{-1}$ ). The potential minimum for $\mathrm{H}_{2}+\mathrm{CO}$ lies $1931 \mathrm{~cm}^{-1}$ above the global minimum for formaldehyde, whereas the zero point energy for $\mathrm{H}_{2}+\mathrm{CO}$ lies $2028 \mathrm{~cm}^{-1}$ below that of formaldehyde. In calculating excitation energies, we take the zero point energy for formaldehyde to be 5848 $\mathrm{cm}^{-1}$, calculated from the harmonic value.

There are many ways to display the formaldehyde potential energy surface. We choose to use the $r_{H}, \theta, \phi$ coordinate system and to display in two-dimensional contour plots the minimum potential for $\mathrm{H}-\mathrm{HCO}$ at any configuration for the other four of the $3 N-6=6$ coordinates. The $\phi, r_{H}$ projection of trajectories provides particularly important information about roaming, while the $\theta, r_{H}$ and $\phi, \theta$ projections also provide useful insight. The minimum potential is determined from the trajectories as follows. For each of the 40204 trajectories that produced reaction either to $\mathrm{H}_{2}+\mathrm{CO}$ or to $\mathrm{H}+\mathrm{HCO}$, we calculated the 
potential for approximately 1-200 frames before the "reaction frame," defined below, to 1100 frames after the "reaction frame." Collectively, these sample a wide range of coordinates. The reaction frame for trajectories producing $\mathrm{H}_{2}+\mathrm{CO}$ was found by tracking the trajectory in reverse from the last frame and then adding two frames to the frame in which both the $\mathrm{H}-\mathrm{H}$ bond had broken $(\mathrm{H}-\mathrm{H}>1.7 \AA)$ and one $\mathrm{C}-\mathrm{H}$ bond had been formed $(\mathrm{C}-\mathrm{H} \leq 1.65 \AA)$. The reaction frame for trajectories producing $\mathrm{H}+\mathrm{HCO}$ was taken to be the frame in which the $\mathrm{H}-\mathrm{H}$ distance exceeded $25.5 \AA$. The minimum potentials were tabulated for a $20 \times 20 \times 20$ grid in the $r_{H}, \theta, \phi$ variables in the ranges $0-10 \AA, 0-\pi, 0-\pi$, under the constraint that one of the $\mathrm{CH}$ bonds was $\leq 1.65 \AA$; that is, that the structure was $\mathrm{H}_{2} \mathrm{CO}$ or $\mathrm{H}+\mathrm{HCO}$ rather than $\mathrm{H}_{2}+\mathrm{CO}$ (which is typically at a lower potential). The resulting minima were used to produce three two-dimensional contours of the minimum potential which are shown in Fig. 1 along with a representative trajectory following the TS route to $\mathrm{H}_{2}+\mathrm{CO}$ products. The low potential energy locations on these contour diagrams correspond to $\mathrm{H}_{2} \mathrm{CO}$ configurations, whereas the high potential energy locations correspond to $\mathrm{H}+\mathrm{HCO}$ configurations. The TS, taken from Zhang et al., ${ }^{14}$ is at $r_{H}=$ $1.978 \AA, \theta=2.250, \phi=0$, but is not easily identified on the PES, for which the lowest bin in $\phi$ is centered near 0.079 radians. The potential surface has a particularly broad and flat plateau region most easily seen in the $\phi, r_{H}$ or $\theta, r_{H}$ contour diagrams. Its potential energy is roughly $33200 \mathrm{~cm}^{-1}$, very near to the dissociation energy to $\mathrm{H}+\mathrm{HCO}, 33239 \mathrm{~cm}^{-1}$.

Individual trajectories were examined to determine those which could be assigned to roaming. The main criterion for roaming was that the trajectory should traverse the high potential energy plateau region in the $\phi, r_{H}$ contour diagram. For $\mathrm{H}_{2}+\mathrm{CO}$ products, a strategy for distinguishing the roaming trajectories from the much more frequent TS trajectories was to order all unassigned trajectories according to one of the evaluated properties (Table 3) and then to individually examine those trajectories whose property value was most associated with roaming. Properties that indicated roaming included, in order of preference, 1) large endpoint $\mathrm{H}_{2}$ vibrational energy, or 2) large fraction of frames preceding the reaction 

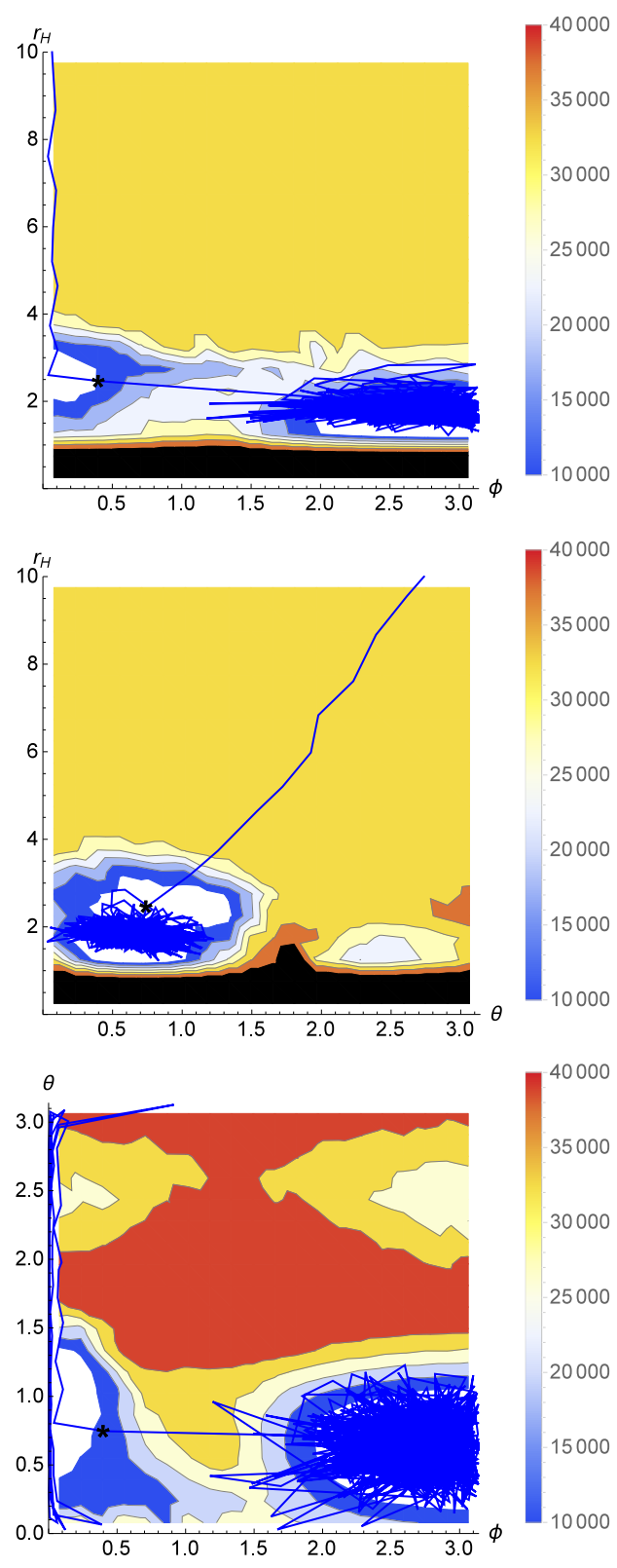

Figure 1: Two-dimensional PES contours in the $r_{H}, \theta, \phi$ coordinate system with a typical TS trajectory for $E_{t o t}=36223 \mathrm{~cm}^{-1}$ superimposed in blue. The black $*$ shows the coordinates at the reaction frame. The equilibrium position of formaldehyde is near $r_{H}, \theta, \phi \approx 1.6,0.6,3.14$ The wells at $r_{H}, \theta, \phi \approx 1.6,2.5,0$ or $1.6,2.5,3.14$ are the cis- and trans-CHOH isomers, respectively. White contours next to dark blue ones indicate that the potential is less than $10000 \mathrm{~cm}^{-1}$. The units of $r_{H,} \phi$, and $\theta$ are in $\AA$, radians, and radians, respectively. 
frame in which a) $\phi>.6$ and $r_{H}>.74 \phi+2.1$, (these values are near the boundary of the plateau region of the potential) b) the average $\mathrm{KE}$ was low, c) the average number of $\mathrm{CH}$ bonds $(\mathrm{C}-\mathrm{H} \leq 1.65 \AA$ ) was closer to one than two, and $\mathrm{d}$ ) the average $\mathrm{H}-\mathrm{H}$ bond length was large. Using these criteria, an examination of a set of 200-300 trajectories at each energy range (of a total of ca. 2500) identified nearly all of those that exhibited roaming just prior to reaction to form molecular products. Identification of those trajectories that roam but then return to the $\mathrm{H}_{2} \mathrm{CO}$ well before reacting over the TS pathway, as well as identification of those trajectories that roam before producing radical products, was performed using criterion 2a) evaluated for either the entire trajectory or for the 50 frames before the radical reaction frame, respectively.

A typical trajectory that exhibits roaming before reaction to molecular products is plotted on the $\phi, r_{H}$ contour diagram in Fig. 2, where the total excitation energy is $36223 \mathrm{~cm}^{-1}$; all three two-dimensional projections of this trajectory are shown in Fig. S1 of the supporting information. Note that much of the trajectory after it leaves the $\mathrm{H}_{2} \mathrm{CO}$ well is in the plateau region. Each trajectory could also be investigated by plotting the number of each type of bond, the bond distances, the bond angles, the coordinates $r_{H}, \theta, \phi$, the angular momentum for each atom, and the kinetic energy as a function of trajectory frame (time). It became clear from examining these plots that the reaction frame for roaming trajectories leading to molecular products was preceded by periods in which the criteria 2a)-2d) were fulfilled. A further feature in many roaming trajectories was the appearance of nearly straight lines in the $r_{H}, \phi$ plane, indicating nearly free azimuthal rotation of the roaming hydrogen atom around the $\mathrm{CO}$ axis. An extreme example of this behavior is shown by the time-domain trajectory of Figure 3, where the total excitation energy is $35000 \mathrm{~cm}^{-1}$. The very regular lines describing $\phi$ as a function of time in this figure indicate that the roaming hydrogen atom rotates uniformly around the $\mathrm{CO}$ axis more than 15 times before eventually reacting to give molecular products. Note that for most of the roaming period, the value of $r_{H}$ is more than $7 \AA$ and that there is simultaneous rotation in the polar angle, $\theta$. This trajectory may 
also be viewed as projections onto pairs of the $r_{H}, \theta, \phi$ coordinates, as shown in Fig. S2. Although this trajectory is unusual, it is by no means an isolated example. Figure S3 shows several more trajectories with long roaming times, while Fig. S4 shows several with short ones. Average roaming times are listed in line 46 of Tables 3 and S1.

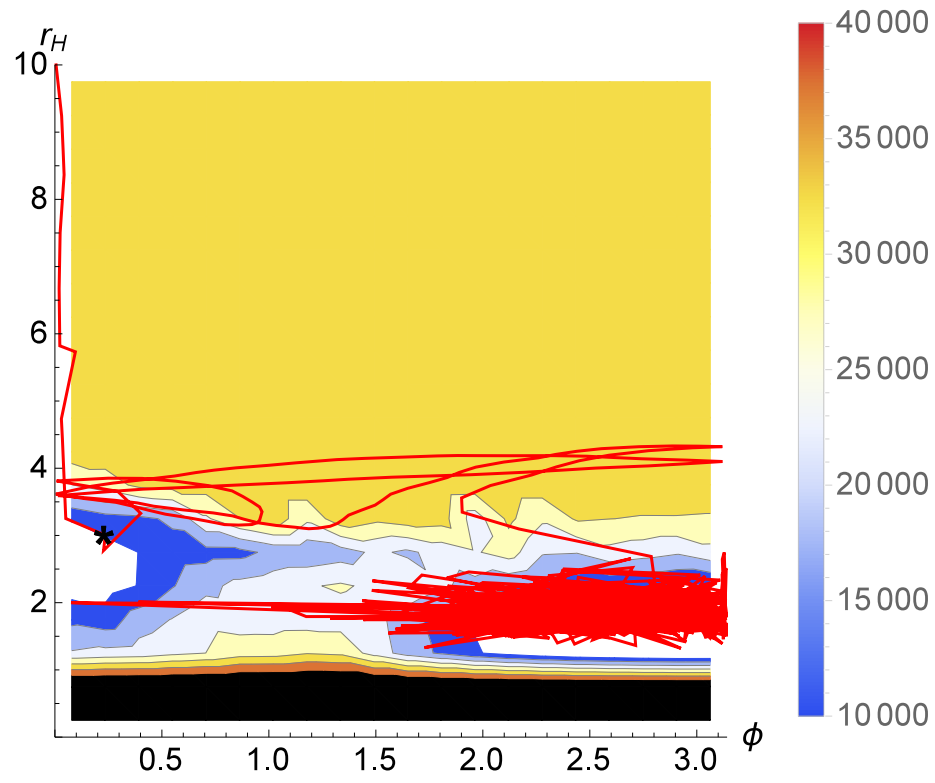

Figure 2: Roaming trajectory at Etot $=36223 \mathrm{~cm}^{-1}$. Formaldehyde almost dissociates via the TS route but eventually roams before reacting at the configuration given by the black *. The nearly straight lines in the high-potential region indicate nearly free rotation of the roaming $\mathrm{H}$ atom about the $\mathrm{CO}$ axis. The units or $r_{H}$ and $\phi$ are $\AA$ and radians, respectively.

\section{Flux Maps}

As a method for investigating the differences between roaming and TS trajectories, we constructed flux maps projected onto pairs of the $r_{H}, \theta, \phi$ coordinates. These were obtained by tabulating 1) the values of the coordinates for each frame near the reaction frame, 2) the change in these values at each frame based on adjacent frames, and 3) the potential energy at each frame. The last of these was used to construct the "minimum energy" potential described in the previous section. The former two were used to construct the flux diagram, giving, on the basis of a $10 \times 10 \times 10$ grid, the average flux of trajectories passing through each bin of pairs in $r_{H}, \theta, \phi$ space. The arrow angles give the direction of the flux, while the 

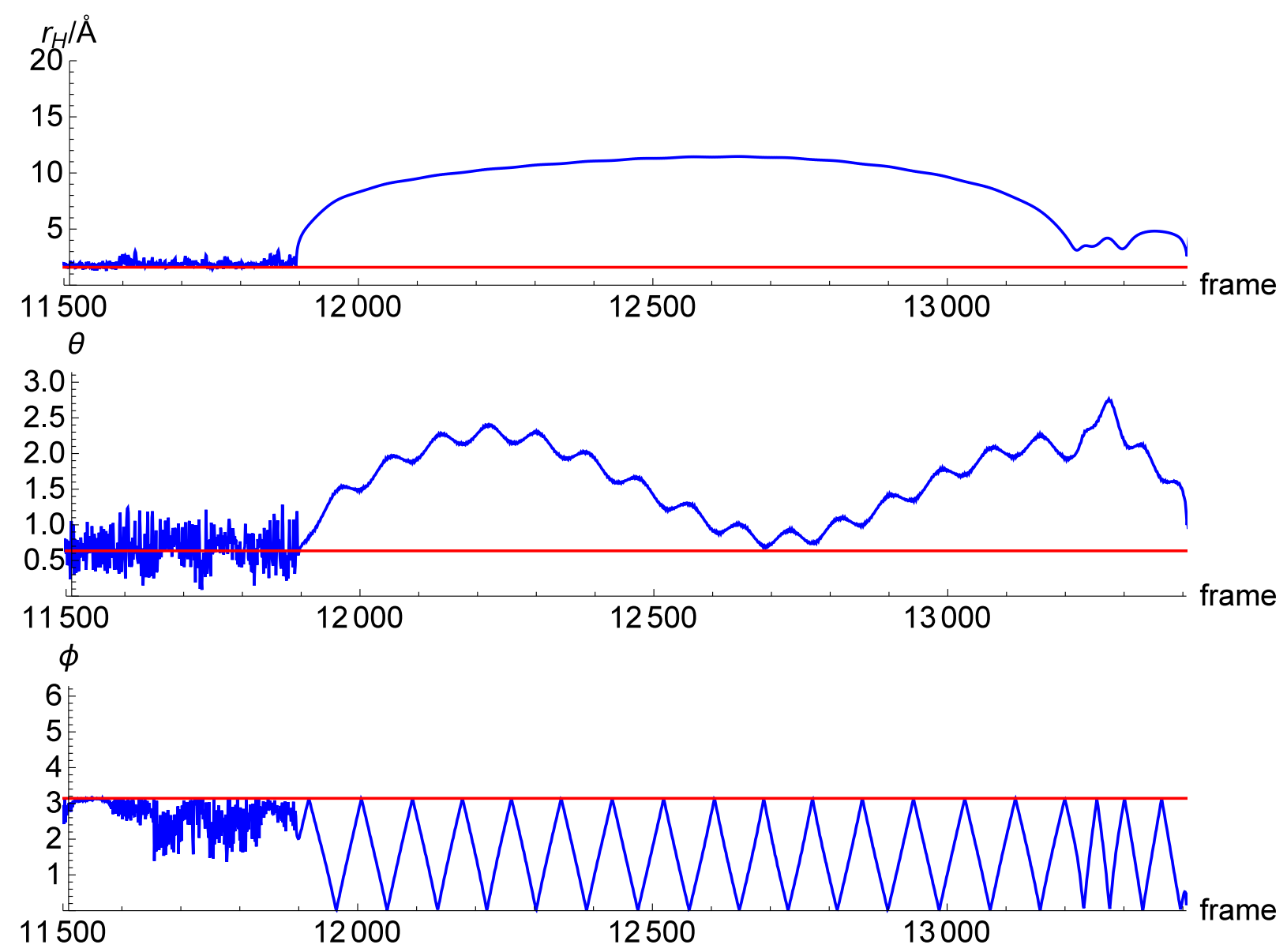

Figure 3: An example of roaming showing $>15$ cycles of rotation of the roaming $\mathrm{H}$ atom around the CO axis. The red line shows the initial value of the coordinate. 
arrow length is proportional to the magnitude. Flux maps projected onto the $r_{H}, \phi$ minimum potential energy contours for TS reactions to $\mathrm{H}_{2}+\mathrm{CO}$ (blue), for roaming reactions to $\mathrm{H}_{2}$ $+\mathrm{CO}$ (red), and for reaction to $\mathrm{H}+\mathrm{HCO}$ (green) are shown in Fig. 4. These and other coordinate pair projections are also shown in Figs. S5-S7. The arrow lengths within a given figure are normalized, but the length of an arrow in one of these figures compared to length in another needs further normalization by the number of trajectories that follow the TS, roaming, or radical reaction route. These numbers are given in Table 2.

\section{Reaction Configuration Plots}

The tabulations that were used to form the minimum energy PES and the flux maps were also useful in characterizing the reaction configurations for TS and roaming trajectories leading to molecular products. As described previously, the "reaction frame" for trajectories producing $\mathrm{H}_{2}+\mathrm{CO}$ was found by tracking the trajectory in reverse and then adding two frames to the frame in which both the $\mathrm{H}-\mathrm{H}$ bond had disappeared $(\mathrm{H}-\mathrm{H}>1.7 \AA)$ and one $\mathrm{C}-\mathrm{H}$ bond had been formed $(\mathrm{C}-\mathrm{H} \leq 1.65 \AA)$. We then tabulated the reaction configuration in $r_{H}, \theta, \phi$ coordinates and plotted it for each trajectory as a point on each of the twodimensional projections. Figure 5 shows such a plot in the $r_{H}, \phi$ plane for a total energy of $35000 \mathrm{~cm}^{-1}$. The red dots are for trajectories that were identified as roaming, whereas the blue dots are for trajectories following the TS route to products. It is clear that there is a substantial difference in how the reaction takes place, with the roaming configuration taking place at larger $r_{H}$ and smaller $\phi$ than the TS configuration. Similar plots for all three two-dimensional projections are shown in Fig. S8. The average configurations for different excitation energies are provided in lines 43 and 44 of Tables 3 and S1. In general, as the excitation energy increases from 34500 to $41010 \mathrm{~cm}^{-1}$, the reaction frame average $r_{H}$ for roaming increases from 2.8 to $3.1 \AA$, whereas the average $r_{H}$ for the TS reaction increases from 2.10 to $2.40 \AA$. The $\phi$ values do not change substantially, but $\phi$ for the roaming reaction (about $0.4-0.5)$ is less somewhat than that for the TS reaction (0.5-0.6). 

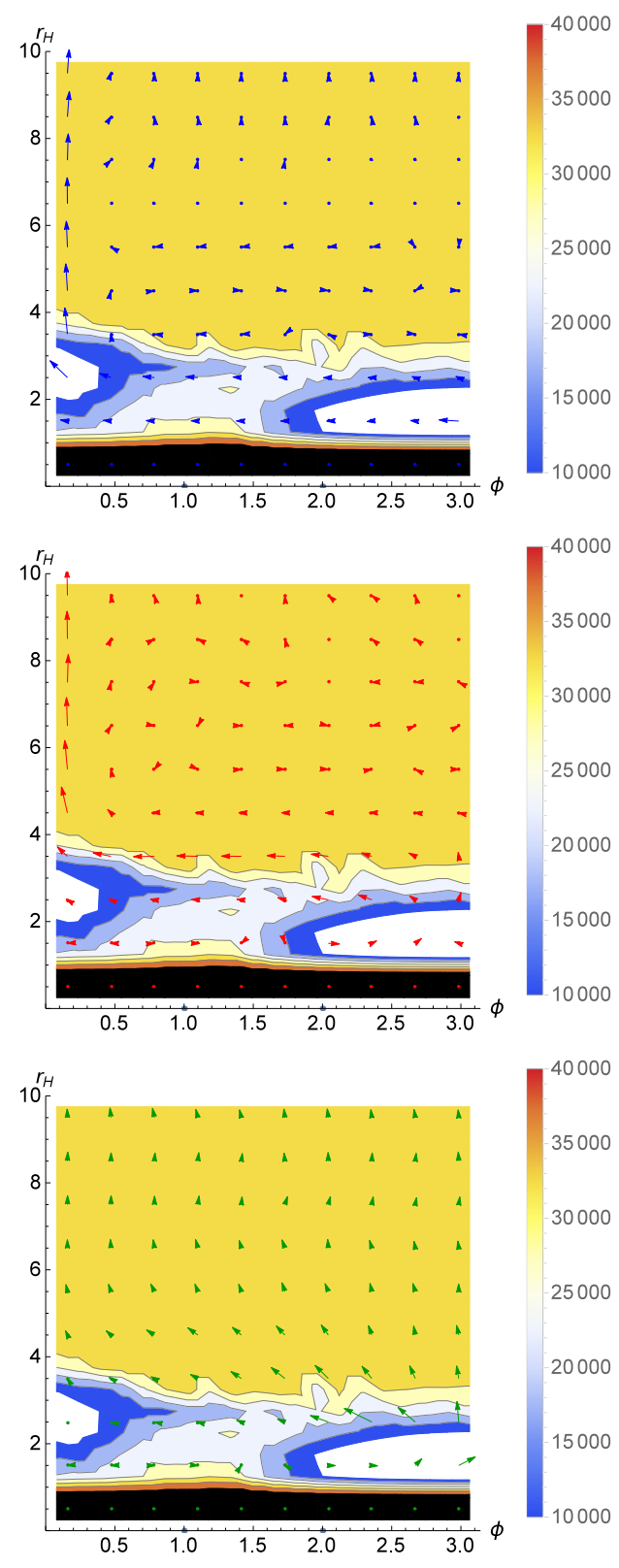

Figure 4: Flux maps for TS reactions to $\mathrm{H}_{2}+\mathrm{CO}$ (top), roaming reactions to $\mathrm{H}_{2}+\mathrm{CO}$ (middle), and reactions to $\mathrm{H}+\mathrm{HCO}$ (bottom). All maps are for an excitation energy of $35000 \mathrm{~cm}^{-1}$. The units of $r_{H,}$, , and $\theta$ are in $\AA$, radians, and radians, respectively. 


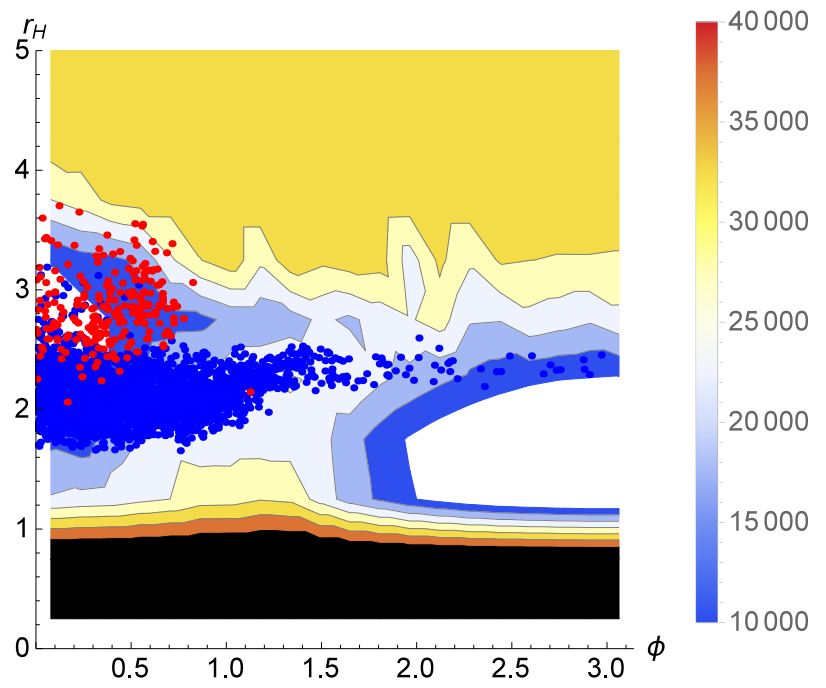

Figure 5: Reaction configuration plot for TS (blue, $n=2643$ ) and roaming (red, $n=233$ ) reactions at $E_{\text {tot }}=35000 \mathrm{~cm}^{-1}$ projected onto the $r_{H}, \phi$ plane, superimposed on the minimum energy potential surface contour plot. The units or $r_{H}$ and $\phi$ are $\AA$ and radians, respectively.

A plot of three representative frames of molecular configurations containing the reaction frame is shown in Fig 6 for TS (top) and for roaming (bottom) trajectories. In the TS sequence, the $\mathrm{H}-\mathrm{H}$ bond is made while both $\mathrm{H}$ atoms are close to the carbon atom, whereas in the roaming sequence, the $\mathrm{H}-\mathrm{H}$ bond is formed when both $\mathrm{H}$ atoms are relatively far from the carbon atom. Note that in the roaming structure the $\mathrm{C}-\mathrm{H}-\mathrm{H}$ bond angle is approximately $180^{\circ}$, whereas in the TS structure this angle is $90^{\circ}$ or less. These observations will be of use to us in determining the nature of roaming in the Discussion below. 

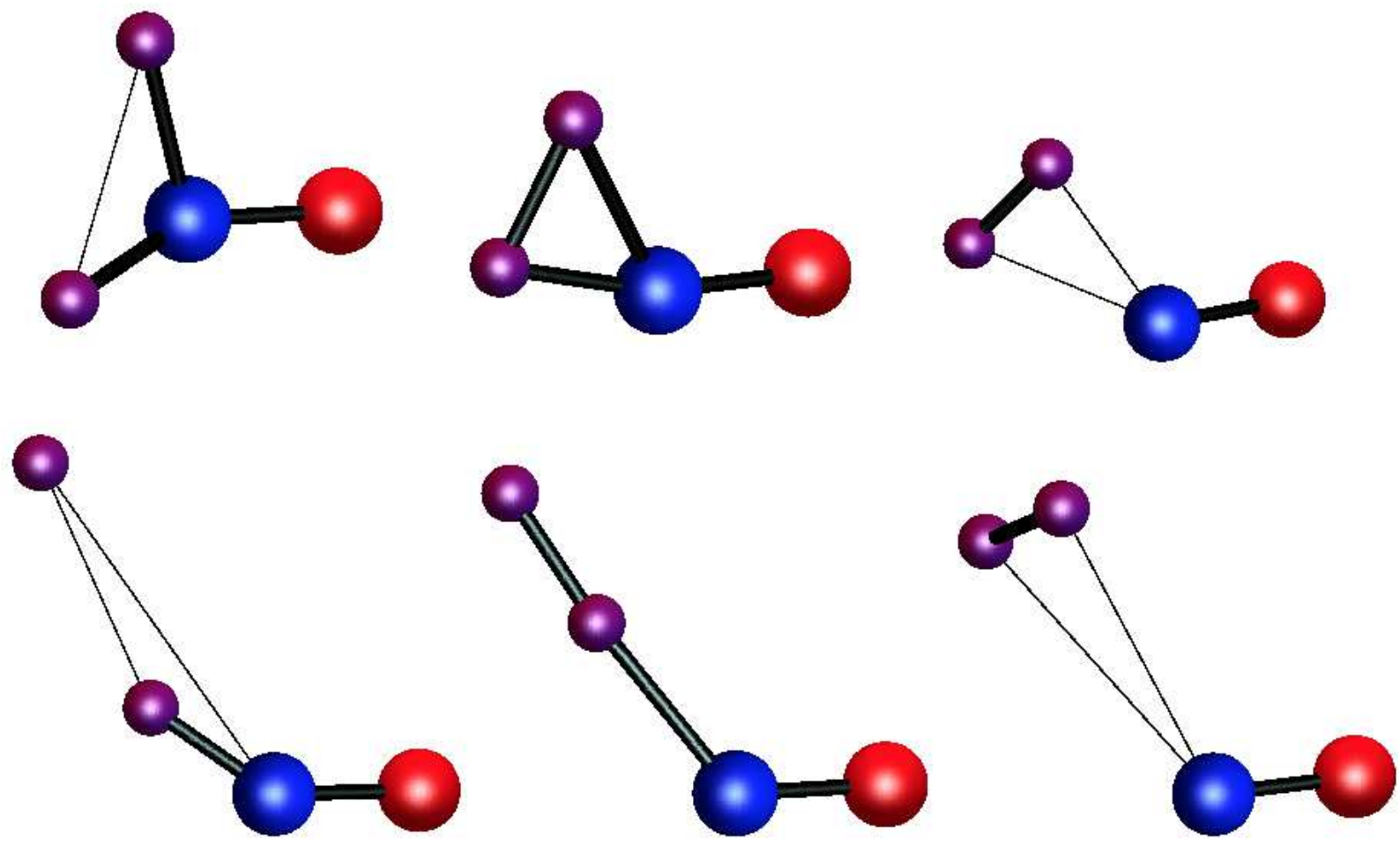

Figure 6: top, left to right: three consecutive frames of structures including the reaction frame (center) for a representative TS trajectory. bottom, left to right: three consecutive frames of structures including the reaction frame (center) for a representative roaming trajectory. 


\section{Discussion}

\section{Comparison to experiment and correlations associated with roaming}

Roaming in the photodissociation of formaldehyde was discovered because of the unusual correlations between the rotational excitation of $\mathrm{CO}$ and the vibrational excitation of $\mathrm{H}_{2}$. In product imaging experiments, low rotational levels of $\mathrm{CO}$ were found to have low velocities, indicating that the vibrational energy of $\mathrm{H}_{2}$ was very high. In contrast, higher rotational levels of $\mathrm{CO}$ were found to have higher velocities, consistent with lower vibrational excitation of the $\mathrm{H}_{2} \cdot{ }^{8-10}$ These two sets of correlated products indicated that the dissociation might be taking place via two different reaction mechanisms, and both experimental and trajectory studies identified roaming as an alternative mechanism to the previously known transition state mechanism. ${ }^{8-16}$ Furthermore, trajectories performed on the same potential energy surface ${ }^{14}$ that has been used here were found to produce good agreement with these experimentally determined correlations. Although more detail is available from the current trajectories concerning the properties of the dissociation, the differences between the two reaction channels, and the energy dependence, there are, to date, few new published experimental results that provide additional points of comparison. On the other hand, there are forthcoming papers that do provide more experimental detail about the dissociation of formaldehyde, ${ }^{24}$ and comparisons with the current trajectory results are underway. In addition, work is proceeding on a new and more accurate potential energy surface, so one may anticipate that substantially more detail will soon be available.

We do note one aspect of our trajectory analysis that has not often been included elsewhere - vector correlations. Tables 3 and S1 (lines 21-32) list some of these properties, while Table 4 in columns 2 and 3 lists the average of these quantities over the nine energies studied. In general, we find a propensity for the rotation vectors of both $\mathrm{CO}$ and $\mathrm{H}_{2}$ to be perpendicular to the relative velocity vector, $\left(\beta_{0}^{0}(22) \rightarrow-0.5\right)$, with the correlation being a bit stronger for $\mathrm{CO}$ than for $\mathrm{H}_{2}$ and slightly stronger for the TS trajectories than for the 
roaming ones. This observation is consistent with a planar geometry for both reactions. The values of the dot product between the $\mathrm{CO}$ and $\mathrm{H}_{2}$ rotational unit vectors are slightly positive for the TS trajectories and slightly negative for the roaming ones, implying that there is a slight tendency for co-rotation in the former and for counter-rotation in the latter. At all energies, the values for the correlation between the transition dipole moment (which is in the plane of formaldehyde, perpendicular to the $\mathrm{CO}$ axis) and the relative velocity are close to $\beta=0$, indicating a nearly anisotropic distribution for both TS and roaming trajectories. That the values of $A_{0}^{(2)}=(2 / 5) P_{2}\left(\cos \theta_{j}\right)$, where $\theta_{j}=$ angle between the product rotational vector and transition dipole, are also close to zero indicates that there is practically no alignment of the $\mathrm{CO}$ and $\mathrm{H}_{2}$ rotational vectors with respect to the transition dipole moment.

Chang, Minichino, and Miller ${ }^{65}$ used an empirical valence-bond (EVB) approach to develop a potential for the dissociation of formaldehyde. Trajectories starting near the region of the known transition state and using the best version of the EVB potential produced results which were in agreement with the most recent experimental work at the time on the translational energy distribution and the $\mathrm{H}_{2}$ vibrational and rotational distributions. The authors also published plots of the distribution of several angles, from which vector correlation parameters can be derived. More recently, Tsai and $\operatorname{Lin}^{66,67}$ developed a multicenter impulsive model to provide insight into the TS and roaming channels. The second of these papers specifically compares the vector properties of the model to the results from Chang, Minichino, and Miller. For the TS, the impulsive model and the earlier trajectory results were in good agreement. Tsai and Lin also applied the model to roaming, and made predictions of angular distributions for this channel. Neither Chang, Minichino, and Miller nor Tsai and Lin tabulated values of the vector correlation parameters shown in column 1 of Table 4, but it is possible from the diagrams to estimate them fairly accurately using a weighted sum of the properties at the average angles and those \pm .5 FWHM on either side. These values are tabulated in column three of Table 4 for the TS trajectories and impulse model and in column 4 for the roaming impulse model. 
From Table 4 we can compare our results to those of these previous authors and gain some insight into whether vector correlations can help to identify roaming in formaldehyde. We see that the $\beta_{0}^{0}(22)$ values are similar except that the value in the impulsive model for roaming is somewhat lower than the value from the current trajectories. The current trajectories suggest that there is a small difference between TS and roaming values, but it would be difficult experimentally to distinguish the two mechanisms on this basis. The current trajectory values for $\beta$ and $A_{0}^{(2)}$ are all so small that these parameters cannot be used to distinguish TS and roaming either. The previous trajectories and the impulsive model predict somewhat larger values, both of these two parameters and of the dot product of the rotational vectors. Both our results and the previous ones suggest that there is a measurable difference in the dot product of unit rotational vectors for TS trajectories vs roaming ones.

Table 4: Vector Correlations in $\mathrm{H}_{2} \mathrm{CO}$ Dissociation

\begin{tabular}{|c|c|c|c|c|}
\hline Property & Traj TS* & Traj $\mathrm{R}^{*}$ & Lit TS† $\ddagger$ & Lit R $\ddagger$ \\
\hline \hline$\beta_{0}^{0}(22)(\mathrm{CO})$ & -0.50 & -0.45 & -0.49 & -0.06 \\
\hline$\beta_{0}^{0}(22)\left(\mathrm{CH}_{2}\right)$ & -0.25 & -0.37 & -0.31 & -0.13 \\
\hline$\beta$ & -0.025 & -0.091 & 0.75 & -0.38 \\
\hline$A_{0}^{(2)}(\mathrm{CO})$ & 0.039 & 0.038 & -0.20 & -0.05 \\
\hline$A_{0}^{(2)}\left(\mathrm{H}_{2}\right)$ & -0.013 & -0.013 & -0.016 & -0.05 \\
\hline $\mathbf{J}(\mathbf{C O}) \bullet \mathbf{J}\left(\mathbf{H}_{\mathbf{2}}\right)$ & 0.16 & -0.16 & 0.86 & 0. \\
\hline
\end{tabular}

Our trajectory results agree qualitatively with the strong product state correlations previously reported, which is not surprising since they were run on the same surface with the same methods. One way to demonstrate these correlations is by plotting a distribution of points correlating the internal energy of $\mathrm{CO}$ with that of $\mathrm{H}_{2}$. An example is show in Fig. 7 for an excitation energy of $35500 \mathrm{~cm}^{-1}$. Similar figures for all nine excitation energies are shown in Fig. S9. As can be seen immediately from these figures, there is substantial separation between those points associated with roaming trajectories (red) and those associated with TS trajectories (blue), particularly at lower excitation energies. The separation becomes a bit less pronounced at the highest energies, where the differences between roaming properties 
and TS ones start to diminish, but it is still true that the roaming channel has very high $\mathrm{H}_{2}$ internal excitation.

Finally, we note that the vibrational distribution of the $\mathrm{H}_{2}$ product from our roaming trajectories is similar to both that from the experiments and that found for the $\mathrm{H}+\mathrm{HCO}$ reaction, studied in trajectories on the same surface by Christoffel and Bowman. ${ }^{25}$ These authors identified three mechanisms for $\mathrm{H}+\mathrm{HCO}$ reaction to $\mathrm{H}_{2}+\mathrm{CO}$ : direct abstraction, roaming followed by reaction, and entrance into the $\mathrm{H}_{2} \mathrm{CO}$ well followed by TS reaction. The former two of these mechanisms had similar $\mathrm{H}_{2}$ vibrational distributions, although of different total cross section, and these distributions are similar to that found here for roaming. The authors did not report a reaction configuration for either of these mechanisms, but it seems likely to be similar to that for our roaming trajectories, shown in the lower half of Fig. 6 .

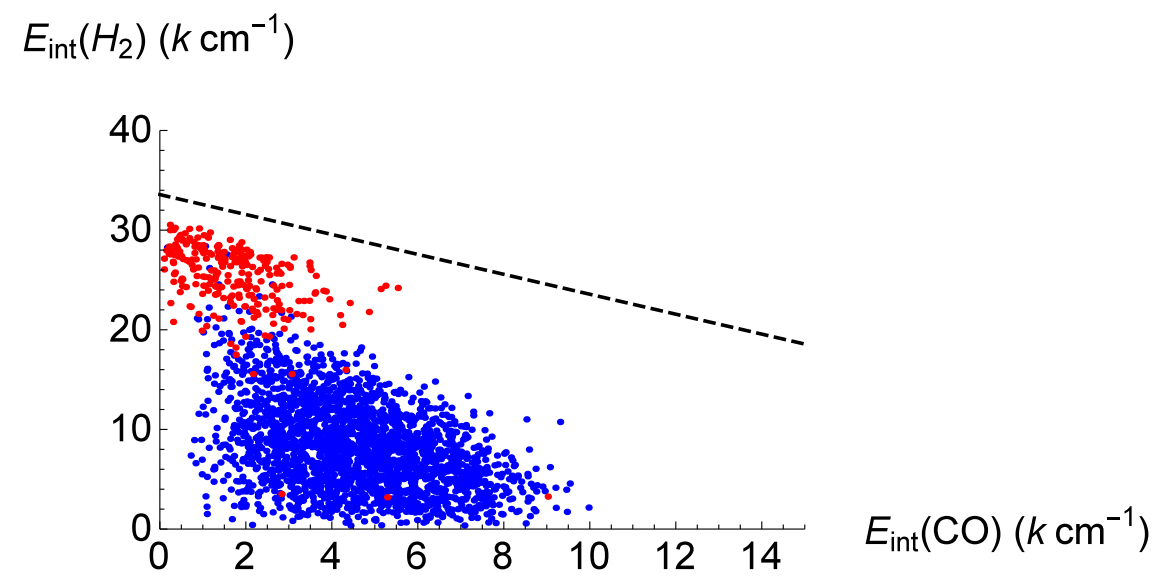

Figure 7: Correlation between $E_{\text {int }}\left(\mathrm{H}_{2}\right)$ and $E_{\text {int }}(\mathrm{CO})$ for an excitation energy of $35500 \mathrm{~cm}^{-1}$. Red points $(n=257)$ are for roaming trajectories, whereas blue points $(n=2374)$ are for TS trajectories. The distance between any point and the dashed line gives the sum of the energy associated with orbital motion of the $\mathrm{CO}$ and $\mathrm{H}_{2}$ and the energy in relative translation of these fragments.

\section{The nature of roaming}

The trajectory paths projected onto the $r_{H}, \theta, \phi$ coordinates, the flux diagrams, and the reaction coordinate information allow a detailed picture of the roaming mechanism. Although roaming follows trajectories that are far from the minimum energy path, the general nature 
of a reaction via roaming and via the TS is similar. In both cases, the $\phi$ coordinate, initially at $\phi=\pi$, decreases, bringing the $\mathrm{H}$ atoms toward one another. Because the $r_{H}$ coordinate always indicates the distance from the $\mathrm{CO}$ center of mass to the more distant $\mathrm{H}$ atom, the trajectory projections onto the $r_{H}, \theta, \phi$ coordinates cannot, by themselves, distinguish the position of the $\mathrm{H}$ atom that has the shorter distance. However, the average reaction coordinates, given in lines 43 and 44 of Tables 3 and S1, show that the roaming reaction has an $r_{H}$ coordinate significantly larger than does the TS reaction. This difference may also be seen by comparing the flux diagrams for the TS (blue) and roaming (red) trajectories, as shown in Fig. 8. The major flux for the TS trajectories occurs as $\phi$ decreases with $r_{H}$ near $2.5 \AA$, whereas that for the roaming trajectories occurs with $r_{H}$ near $3.5 \AA$. In addition, we see from Fig. 5 that in the TS pathway, the $\mathrm{H}-\mathrm{H}$ bond is made while the $\mathrm{C}-\mathrm{H}$ bonds are still rather short, whereas in the roaming pathway, the $\mathrm{H}-\mathrm{H}$ bond is made when both $\mathrm{C}-\mathrm{H}$ bonds are elongated, if not broken. The roaming sequence also suggests why the $\mathrm{H}_{2}$ vibrational energy is so high: the $\mathrm{H}$ atom closer to the carbon moves a very substantial distance during the reaction. In both pathways, $\theta$ remains nearly constant while $\phi$ decreases substantially. In the TS case, there is a very sudden change in $\phi$ as the $\mathrm{H}$ atom crosses the $\mathrm{CO}$ axis between the first and second frames shown. In contrast, for the roaming reaction, $\phi$ has diminished nearly to zero long before the reaction takes place. Roaming reactions are characterized by an elongation of $r_{H}$ and a decrease from $\phi=\pi$ toward $\phi=0$, whereas the TS pathway is characterized by much less change in $r_{H}$ as $\phi$ decreases. In order for $r_{H}$ to increase in the roaming trajectory, most of the KE of the system must be converted to surmount the potential energy of the plateau region of the PES. If the value of $r_{H}$ is near $2.8-3.1 \AA$ when $\phi \rightarrow 0$ with $\theta \approx 0.8-0.9$, then the roaming trajectory will react to give $\mathrm{H}_{2}+\mathrm{CO}$, and because the $\mathrm{H}-\mathrm{H}$ bond at that point is elongated, the reaction will produce substantial $\mathrm{H}_{2}$ vibration. On the other hand, if $r_{H}$ is somewhat larger than $3.1 \AA$ as $\phi \rightarrow 0$, or if $\theta>1$, the $\mathrm{H}$ will continue to rotate around the $\mathrm{CO}$ axis without reaction. If enough $\mathrm{KE}$ is available to the $r_{H}$ coordinate, the system will react to $\mathrm{H}+\mathrm{HCO}$, but if not, the $r_{H}$ coordinate will oscillate 
slowly until one of two outcomes occurs. The value of $r_{H}$ may become short enough near $\phi \rightarrow \pi$ to return the system to the $\mathrm{H}_{2} \mathrm{CO}$ well, or it may approach $2.8-3.1 \AA$ as $\phi \rightarrow 0$, in which case it will abstract the shorter $\mathrm{H}$ atom to form $\mathrm{H}_{2}+\mathrm{CO}$. It is now apparent that the straight-line trajectories seen in $r_{H}, \phi$ projections such as those in Figs. 2, S2 (top), and S3 can be understood as a consequence of the reaction requirements that $\phi$ should decrease and that the $\mathrm{H}$ atoms should have a configuration such as those shown in the bottom sequence of Fig. 6. If the $r_{H}$ coordinate is too long, free internal rotation occurs until the phasing is correct for either reaction or return to the $\mathrm{H}_{2} \mathrm{CO}$ well.

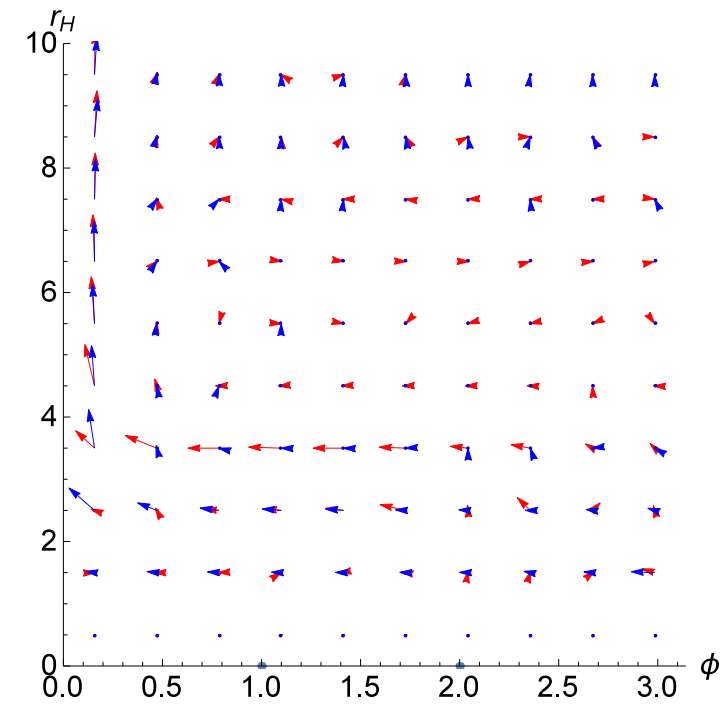

Figure 8: Comparison of TS (blue) and Roaming (red) Flux Maps for $E_{t o t}=36223 \mathrm{~cm}^{-1}$. The magnitudes of the two fluxes are not normalized relative to one another; if they were, all red arrows would be a factor of (1/0.098) times as long (see line 4 of Table 2 ). The units or $r_{H}$ and $\phi$ are $\AA$ and radians, respectively.

There is some variation during the roaming trajectory in the $\theta$ coordinate, as can be seen in Fig. 3 and S2. Mauguiere et al. ${ }^{22}$ have studied a reduced-dimensionality system for formaldehyde that includes only the distance from the $\mathrm{COM}$ of $\mathrm{HCO}$ to the roaming $\mathrm{H}$ atom, $R$, and the angle $\theta$ between the $\mathrm{CO}$ axis and $R$. In this reduced system, roaming was found to occur along unstable periodic orbits involving large changes in $\theta$. Our own work looks strikingly similar, in that the roaming trajectories are easily distinguished on a plot involving the distance to the $\mathrm{H}$ atom and an angle. However, in our study the angle most 
important is $\phi$ rather than $\theta$. Mauguire et al. would not have observed this motion, since the $\phi$ coordinate was not included in their reduced-dimensionality system. Nonetheless, their idea that roaming is due to unstable periodic orbits controlled by phase space constructs is likely to be true.

\section{Kinetic Model for Roaming}

Several kinetic or statistical models have been proposed to describe roaming, ${ }^{54,68,69}$ as reviewed previously. ${ }^{54}$ Klippenstein, Georgievskii and Harding ${ }^{68}$ proposed a five-state kinetic model that included the species formaldehyde, radical products and molecular products as well as two intermediates, a weakly interacting intermediate formed by partial dissociation of a $\mathrm{CH}$ bond and another in which the weakly interacting $\mathrm{H}$ atom is closest to the other $\mathrm{H}$. In all, there are seven rate constants connecting these species. The steady-state assumption was used to determine branching between roaming and radical channels in various limits, and the best results are within a factor of two of trajectory calculations. Andrews, Kable and Jordan ${ }^{69}$ proposed a phase space theory for roaming reactions that depends on the difference in threshold energies for roaming and radical production and on $P_{\text {roam }}$, a probability that phase space states that may roam do so rather than recombining to form reactants. For formaldehyde, the difference in threshold energies was taken to be $161 \mathrm{~cm}^{-1}$, while $P_{\text {roam }}$ was set at 0.99 . Using this model, they found that the branching fraction for roaming fell from 1.0 to about 0.15 over the first $1000 \mathrm{~cm}^{-1}$ of energy in excess of the radical threshold. Their model did not predict the fraction of TS to roaming in the formation of $\mathrm{H}_{2}+\mathrm{CO}$. Ulusoy, Stanton and Hernandez have proposed a model for roaming in ketene. ${ }^{70-72}$

We propose a simple model that borrows some elements of each of the above and provides a fit to the data in Tables 2 and 3. The rate constants ultimately will be determined from the quasi-classical trajectory results, but the model provides a simple way to understand them. It invokes the three species - formaldehyde, bimolecular products and radical products but has only one intermediate, a roaming state, $\mathrm{R}$, that may produce radicals, bimolecular 
products, or decay back to formaldehyde. The model consists of the following steps and associated rate constants:

$$
\begin{aligned}
\mathrm{H}_{2} \mathrm{CO} \longrightarrow \mathrm{R} & k_{R} \\
\mathrm{R} \rightarrow \mathrm{H}_{2} \mathrm{CO} & k_{-R} \\
\mathrm{H}_{2} \mathrm{CO} \longrightarrow \mathrm{H}_{2}+\mathrm{CO} & k_{T S-B i} \\
\mathrm{R} \longrightarrow \mathrm{H}+\mathrm{HCO} & k_{R-R a d} \\
\mathrm{R} \longrightarrow \mathrm{H}_{2}+\mathrm{CO} & k_{R-B i}
\end{aligned}
$$

In these equations, $\mathrm{R}$ represents $\mathrm{H}-\mathrm{HCO}$ in a roaming state; we treat this state as if it were ahighly fluxioinal isomer of formaldehyde. It can be formed from formaldehyde with rate constant $k_{R}$ and can decay back to formaldehyde with rate constant $k_{-R}$. Formaldehyde can react to molecular products directly via the transition state with rate constant $k_{T S-B i}$, or it can also reach these products from $\mathrm{R}$ with rate constant $k_{R-B i}$. The state $\mathrm{R}$ may also decompose to radical products with rate constant $k_{R-R a d}$. Because $\mathrm{R}$ disappears very quickly compared to the rate of the overall disappearance of $\mathrm{H}_{2} \mathrm{CO}$, it is appropriate to treat it as being in steady-state, as used previously; ${ }^{68}$ i.e., we set $d[\mathrm{R}] / d t=0$. In this approximation it is easy to show that $[\mathrm{R}]=k_{R}\left[\mathrm{H}_{2} \mathrm{CO}\right] / X$, where $X=\left(k_{-R}+k_{R-R a d}+k_{R-B i}\right)$. Furthermore, $F R$, defined as the ratio of roaming production of bimolecular products to the total rate of bimolecular production, is given by $F R=\left(k_{R-B i} k_{R} / X\right) /\left(k_{T S-B i}+\left(k_{R-B i} k_{R} / X\right)\right)$. The ratio FRad, defined as the ratio of total radical to total bimolecular products, is $F R a d=$ $\left(k_{R-R a d} k_{R} / X\right) /\left(k_{T S-B i}+\left(k_{R} k_{R-B i} / X\right)\right)$. TDRC the total decomposition rate constant of formaldehyde is given by $T D R C=\left(k_{T S-B i}+k_{R}\right)-k_{-R} k_{R} / X$. Thus, there are five rate constants and the following four observables: TDRC, obtained from line 45 of Tables 3 and S1; FRad, obtained from ratio of line 3 to line 2 of Table 2; FR, obtained from line 4 of Table 2; and $k_{R-B i}$, obtained from the reciprocal of line 46 of Tables 3 and S1. Although 
this system of five unknowns and four equations would seem to be unsolvable, we note that there is evidence from line 5 of Table 2 that $k_{-R}$ is much smaller than $k_{R-B i}+k_{R-R a d}$; that is, there are many more trajectories that produce $\mathrm{H}_{2}+\mathrm{CO}$ and $\mathrm{H}+\mathrm{HCO}$ from $\mathrm{R}$ than return to $\mathrm{H}_{2} \mathrm{CO}$. This assumption is similar to taking $P_{\text {roam }}=1.0$, as used previously. ${ }^{69}$ If we neglect $k_{-R}$ relative to $k_{R-B i}+k_{R-R a d}$, we then can solve at each excitation energy for the remaining four rate constants that are consistent with the measured properties. These rate constants are shown in a log plot in Fig. 9.

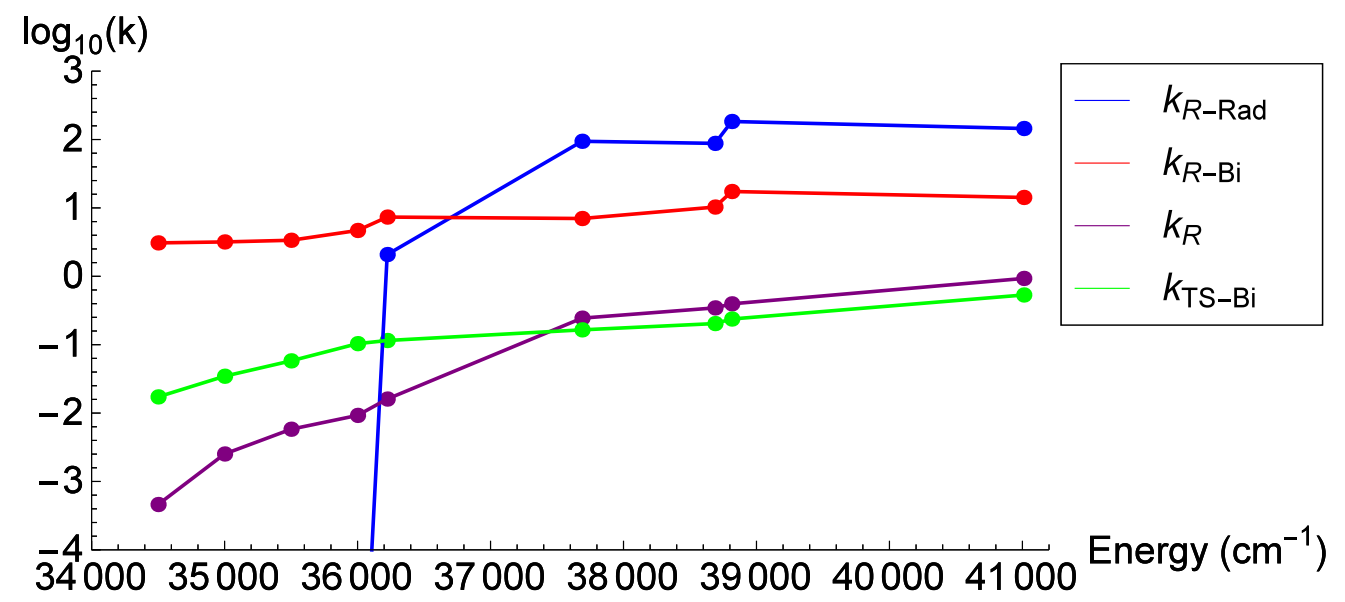

Figure 9: Plot of $\log _{10}$ of the rate constants (in $\mathrm{ps}^{-1}$ ) vs excitation energy for the four rate constants describing a simple model for roaming.

At an excitation energy of $37688 \mathrm{~cm}^{-1}$ and above, all four rate constants are nearly in constant ratio to one another, indicating that the product distributions should be approximately similar. Indeed, except that the roaming fraction increases slightly, the branching fractions are similar, as shown in Table 2. At energies below $37688 \mathrm{~cm}^{-1}$, the rate constants fall off in order of their thresholds: the first to fall off is the rate constant for production of $\mathrm{H}+\mathrm{HCO}$ from $R$ (blue), which has the highest threshold; the next is that for reaching $R$ from $\mathrm{H}_{2} \mathrm{CO}$ (purple); and the last is the rate constant for the TS pathway (green). The rate constant for production of $\mathrm{H}_{2}+\mathrm{CO}$ from $R$ (red) is relatively constant, as might be expected from the fact that reaction to bimolecular products from $\mathrm{R}$ has no apparent barrier; 
trajectories seem to fall into a hole in the plateau before reaction. Thus, the general behavior of the rate constants seems sensible. This view is reinforced by the calculation of RRKM rate constants using sensible data for formaldehyde and $\mathrm{R}$ as well as for the transition states associated with each of the four rate constants. The parameters are listed in Table S3. A comparison of the rate constants calculated using RRKM theory and the rate constants that fit the trajectory data is shown in Figure S10 and demonstrates that it is possible to find transition states that produce rate constants that are very close to those in Fig. 9, above.

We end with a brief discussion of treating roaming as an isomer of formaldehyde. Statistical mechanics shows that equilibrium favors "structures" that have a high density of states at the energy (or temperature) of the system. One situation in which a high density of states is encountered is when the structure has a low energy. Low energy structures will be favored because the density of states increases rapidly with the available energy. In most cases, isomers have such a character. For example, although they do not seem to play a large role in the dissociation, the $\mathrm{CHOH}$ isomer has potential energy wells for both the cis and trans forms, and the density of states is higher in the regions of these structures. A less obvious way for an "isomer" to have a high density of states is not to have a structure at all, or to have one that is extremely loosely defined. In the case of formaldehyde, the high plateau region of the potential energy surface has almost no features that constrain the relationship of the H to the HCO. When the potential "well" is so wide, the energy states are extremely

close together, so that the density of states can be high even though there is neither a well corresponding to a specific structure nor a low energy. It would appear from the applicability of the kinetic scheme above that the formaldehyde roaming state is such an "isomer."

\section{Summary and Conclusions}

The fraction of roaming trajectories leading to $\mathrm{H}_{2}+\mathrm{CO}$ is non-negligible $(2.6 \%)$ even at at the lowest energy studied $\left(34500 \mathrm{~cm}^{-1}\right)$ and generally increases to $13-14 \%$ at the highest 
energy studied $\left(41010 \mathrm{~cm}^{-1}\right)$. Roaming has many characteristic features, but the principal one is that the trajectories enter the plateau region of high potential energy. The duration of roaming decreases with increasing total excitation energy. Long roaming periods are characterized by unstable periodic orbits that involve, mostly, rotation of the distant $\mathrm{H}$ atom around the $\mathrm{CO}$ axis $(\phi)$ or, less commonly, end-to-end rotation $(\theta)$. Short roaming periods involve pathways in the high potential energy region that lead more directly from the $\mathrm{H}_{2} \mathrm{CO}$ well to the configuration for the roaming reaction. This configuration has the roaming atom at a greater distance from the CO COM than the non-roaming hydrogen, but both nearly in the same plane $(\phi \rightarrow 0)$. The reaction configuration is a function of excitation energy. As the energy gets higher, the distinction between roaming and TS trajectories becomes smaller.

Flux analysis shows that the reactions producing $\mathrm{H}_{2}+\mathrm{CO}$ by roaming and by the TS are similar. Both involve rotation of one hydrogen atom by an angle of nearly $\phi=\pi$ followed by reaction. The difference is that in the TS reaction, the rotating atom is still within bonding distance of the carbon atom, whereas for the roaming reaction, the rotating atom is at a distance beyond that of a $\mathrm{CH}$ bond. In the roaming case, if the roaming hydrogen is far enough away, it simply rotates around the $\mathrm{HCO}$ until the $\mathrm{H}$ distance becomes short enough to react. The reaction configuration for roaming, $\left\{r_{H}, \theta, \phi\right\}=\{2.85,0.90,0.40\}$, has a considerably longer $r_{H}$ than the one for the TS reaction, $\{2.20,0.79,0.57\}$, (these data are for an excitation energy of $\left.36000 \mathrm{~cm}^{-1}\right)$.

The branching ratios of products, the total reaction rates, and the roaming times as a function of excitation energy can be predicted by a simple kinetic model in which the roaming is treated as a separate entity on the potential energy surface, much as one might treat an isomer of formaldehyde. Given this observation, it is likely that versions of transition state theory could account for roaming if it is treated as a separate entity. Features of the minimum energy surface provide some guidance as to where minimum recrossing boundaries might be assigned. 


\section{Acknowledgments}

This material is based upon work supported by the Army Research Office (JMB) under award number W911NF-14-1-0208 and by the U.S. Department of Energy, Office of Science, Office of Basic Energy Sciences (RC) under Award Number DE-FG02-97ER14782.

\section{Supporting Information Available}

Supporting information referred to in the text is available, including three tables, and ten figures. This material is available free of charge via the Internet at http://pubs.acs.org

\section{References}

(1) Truhlar, D. G.; Hase, W. L.; Hynes, J. T., Current Status of Transition-State Theory. J. Phys. Chem. 1983, 87, 2664-2682. .

(2) Truhlar, D. G.; Garrett, B. C.; Klippenstein, S. J., Current Status of Transition-State Theory. J. Phys. Chem. 1996, 100, 12771-12800.

(3) Fernandez-Ramos, A.; Miller, J. A.; Klippenstein, S. J.; Truhlar, D. G., Modeling the Kinetics of Bimolecular Reactions. Chem. Rev. 2006, 106, 4518-4584.

(4) Debarre, D.; Lefebvre, M.; Pealat, M.; Taran, J. P. E.; Bamford, D. J.; Moore, C. B., Photofragmentation Dynamics of Formaldehyde: $\mathrm{H}_{2}(\mathrm{v}, \mathrm{J})$ Distributions. J. Chem. Phys. 1985, 83, 4476-4487.

(5) Butenhoff, T. J.; Carleton, K. L.; Moore, C. B., photodissociation dynamics of formaldehyde: $\mathrm{H}_{2}$ rotational distributions and product quantum state correlations. $J$. Chem. Phys. 1990, 92, 377. 
(6) Butenhoff, T. J.; Carleton, K. L.; Zee, R. D. v.; Moore, C. B., The effect of parent rotational state on fragment anisotropy and application to formaldehyde. J. Chem. Phys. 1991, 94, 1947.

(7) Zee, R. D. v.; Foltz, M. F.; Moore, C. B., Evidence for a Second Molecular Channel in the Fragmentation of Formaldehyde. J. Chem. Phys. 1993, 99, 1664-1673.

(8) Townsend, D.; Lahankar, S. A.; Lee, S. K.; Hambreau, C. S. D.; Suits, A. G.; Zhang, X.; Rheinecker, J.; Harding, L. B.; Bowman, J. M., The Roaming Atom: Straying from the Reaction Path in Formaldehyde Decomposition. Science 2004, 306, 1158-1161.

(9) Chambreau, S. D.; Lahankar, S. A.; Suits, A. G., Correlated vH $\mathrm{H}_{2}$ and jCO Product States from Formaldehyde Photodissociation: Dynamics of Molecular Elimination. J. Chem. Phys. 2006, 125, 044302.

(10) Lahankar, S. A.; Chambreau, S. D.; Townsend, D.; Suits, F.; Farnum, J. D.; Zhang, X.; Bowman, J. M.; Suits, A. G., The Roaming Atom Pathway in Formaldehyde Decomposition. J. Chem. Phys. 2006, 125, 044303.

(11) Lahankar, S. A.; Chambreau, S. D.; Zhang, X.; Bowman, J. M.; Suits, A. G., Energy Dependence of the Roaming Atom Pathway in Formaldehyde Decomposition. J. Chem. Phys. 2007, 126, 044314.

(12) Lahankar, S. A.; Goncharov, V.; Suits, F.; Farnum, J. D.; Bowman, J. M.; Suits, A. G., Further Aspects of the Roaming Mechanism in Formaldehyde Dissociation. Chem. Phys. 2008, 347, 288-299.

(13) Goncharov, V.; Lahankar, S. A.; Farnum, J. D.; Bowman, J. M.; Suits, A. G., Roaming Dynamics in Formaldehyde-d $\mathrm{d}_{2}$ Dissociation. J. Phys Chem. A 2009, 113, 15315-15319.

(14) Zhang, X.; Zou, S.; Harding, L. B.; Bowman, J. M., A Global ab Initio Potential Energy Surface for Formaldehyde. J. Phys. Chem. A 2004, 108, 8980-8986. 
(15) Zhang, X.; Rheinecker, J.; Bowman, J. M., Quasiclassical trajectory study of formaldehyde unimolecular dissociation $: \mathrm{H}_{2} \mathrm{CO} \rightarrow \mathrm{H}_{2}+$ CO. J. Chem. Phys. 2005, 122, 114313.

(16) Zhang, X.; Bowman, J. M., New Insights on Reaction Dynamics form Formaldehyde Phototissociation. Phys. Chem. Chem. Phys. 2006, 8, 321-332.

(17) Bowman, J. M., Skirting the Transition State, a New Paradigm in Reaction Rate Theory. Proc. Natl. Acad. Sci. U. S. A. 2006, 103, 16061-16062.

(18) Suits, A. G., Roaming Atoms and Radicals: A New Mechanism in Molecular Dissociation. Acct. Chem. Res. 2008, 41, 873-881.

(19) Bowman, J. M.; Suits, A. G., Roaming Reactions: The Third Way. Phys. Today 2011, $64,33$.

(20) Herath, N.; Suits, A. G., Roaming Radical Reactions. J. Phys. Chem. Lett. 2011, 2, 642-647.

(21) Yin, H. M.; Kable, S. H.; Zhang, X.; Bowman, J. M., Signatures of $\mathrm{H}_{2}$ CO Photodissociation from Two Electronic States. Science 2006, 311, 1443-1446.

(22) Mauguiere, F. A. L.; Collins, P.; Kramer, Z. C.; Carpenter, B. K.; Ezra, G. S.; Farantos, S. C.; Wiggins, S., Phase Space Structures Explain Hydrogen Atom Roaming in Formaldehyde Decomposition. J. Phys. Chem. Lett. 2015, 6, 4123-4128.

(23) Maeda, S.; Taketsugu, T.; Ohno, K.; Morokuma, K., From Roaming Atoms to Hopping Surfaces: Mapping Out Global Reaction Routes in Photochemistry. J. Am. Chem. Soc. 2015, 137, 3433-3445.

(24) Mitchell Stephen Quinn, "The Photodissociation of Small Aldehydes: A Refined Understanding of Prototypical Dynamical Systems," Ph.D. Thesis, University of New South Wales, 2016. 
(25) Christoffel, K. M.; Bowman, J. M., Three Reaction Pathways in the $\mathrm{H}+\mathrm{HCO} \rightarrow \mathrm{H}_{2}+$ CO Reaction. J. Phys. Chem. A 2009, 113, 4138-4144.

(26) Houston, P. L.; Kable, S. H., Photodissociation of Acetaldehyde as a Second Example of the Roaming Mechanism. Proc. Nat. Acad. Sci. U. S. A. 2006, 103, 16079-16082.

(27) Rubio-Lago, L.; Amaral, G. A.; Arregui, A.; Wang, F.; Zaouris, D.; Kitsopoulos, T. N.; Banares, L., Slice Imaging of the Photodissociation of Acetaldehyde. Phys. Chem. Chem. Phys. 2007, 9, 6123-6127.

(28) Shepler, B. C.; Braams, B. J.; Bowman, J. M., Quasiclassical Trajectory Calculations of Acetaldehyde Dissociation on an Global Potential Energy Surface Indicate Significant Non-Transition State Dynamics. J. Phys. Chem. A 2007, 111, 8282-8285.

(29) Haezlewood, B. R.; Jordan, M. J. T.; Kable, S. H.; Selby, T. M.; Osborn, D. L.; Shepler, B. C.; Braams, B. J.; Bowman, J. M., Roaming is the Dominant Mechanism for Molecular Products in Acetaldehyde Photodissociation. Proc. Natl. Acad. Sci. U. S. A. 2008, 105, 12719-12724.

(30) Harding, L. B.; Georgievskii, Y.; Klippenstein, S. J., Roaming Radical Kinetics in the Decomposition of Acetaldehyde. J. Phys. Chem. A 2010, 114, 765-777.

(31) Sivaramakrishnan, R.; Klippenstein, S. J., Direct Observation of Roaming Radicals in the Thermal Decomposition of Acetaldehyde. J. Phys. Chem. A 2010, 114, 755-764.

(32) Lee, K. L. K.; Quinn, M. S.; Maccarone, A. T.; Nauta, K.; Houston, P. L.; Reid, S. A.; Jordan, M. J. T.; Kable, S. H., Two roaming pathways in the photolysis of $\mathrm{CH}_{3} \mathrm{CHO}$ between 328 and 308 nm. Chem. Sci. 2014, 5, 4633-4638.

(33) Li, H.-K.; Tsai, P.-Y.; Hung, K.-C.; Kasai, T.; Lin, K. C., Communication: Photodissociation of $\mathrm{CH}_{3} \mathrm{CHO}$ at $308 \mathrm{~nm}$ : Observationof H-roaming, $\mathrm{CH}_{3}$-roaming, and Transition 
State Pathways Together along the Ground State Surface. J. Chem. Phys. 2015, 142, 041101.

(34) Tsai, P.-Y.; Li, H.-K.; Kasai, T.; Lin, K. C., Roaming as the Dominant Mechanism for Molecular Products in the Photodissociation of Large Aliphatic Aldehydes. Phys. Chem. Chem. Phys. 2015, 17, 23112-23120.

(35) Nakamura, M.; Tsai, P.-Y.; Kasai, T.; Lin, K. C.; Palazzetti, F.; Lombardi, A.; Aquilanti, V., Dynamical, Spectroscopic and Computational Imaging of Bond Breaking in Photodissociation: Roaming and Role of Conical Intersections. Far. Disc. Chem. Soc. 2015, 177, 77-98.

(36) Dhoke, K.; Zanni, M.; Harbola, U.; Venkatraman, R. K.; Arunan, E.; Lin, K. C.; Nenov, A.; Skelton, J.; Miller, R. J. D.; Hirst, J. D.; Aquilanti, V.; Helliwel, J. R.; Keshavamurthy, S.; Ramesh, S.; Ashfold, M. N. R.; Pallipurath, A.; Chowdhury, P. R.; Mukhopadhyay, S.; E., J. E.; Medhi, H.; Goswarni, D.; P., H.; W., J.; Hariharan, M.; Singh, S. K.; Umapathy, S.; Lakshmannam, A.; Nielsen, M. M.; Aravmudhan, S.; Deckert, B.; Ghiggino, K.; Tominaga, K.; Ediwards, A., Dynamics of Chemical Bond: General Discussion. Farad. Disc. Chem. Soc. 2015, 17\%, 121-154.

(37) Tsai, P.-Y.; Chao, M.-H.; Kasai, T.; Lin, K. C.; Lombardi, A.; Palazzetti, F.; Aquilanti, V., Roads Leading to Roam. Role of Triple Fragmentation and of Conical Intersections in Photochemical reactions: Experiments and Theory on Methyl Formate. Phys. Chem. Chem. Phys. 2014, 16, 2854-2865.

(38) Lombardi, A.; Palazzetti, F.; Lin, K. C.; Tsai, P.-Y., Effective Four-center Model for the Photodissociation Dynamics of Methyl Formate. Lect. Notes Computer Sci. 2014, $8579,462-4678$.

(39) Lin, K. C., Regulation of Nonadiabatic Processes in Photolysis of Some Carbonyl Compounds Phys. Chem. Chem. Phys. 2016, accepted, DOI: 10.1039/C5CP07012K. 
(40) Maeda, S.; Ohno, K.; Morokuma, K., A Theoretical Study on the Photodissociation of Acetone: Insight in to the Slow Intersystem Crossing and Exploration of Nonadiabatic Pathways to the Ground State. J. Phys. Chem. Lett. 2010, 1, 1841-1845.

(41) Harding, L. B.; Klippenstein, S. J., Roaming Radical Pathways for the Decomposition of Alkanes. J. Phys. Chem. Lett. 2010, 1, 3016-3020.

(42) Grubb, M. P.; Warter, M. L.; Suits, A. G.; North, S. W., Evidence of Roaming Dynamics and Multiple Channels for Molecular Elimination in $\mathrm{NO}_{3}$ Photolysis. J. Phys. Chem. Lett. 2011, 1, 2455-2458.

(43) Fernando, R.; Dey, A.; Broderick, B. M.; Fu, B.; Homayoon, Z.; Bowman, J. M.; Suits, A. G., Visible/Infrared Dissociation of $\mathrm{NO}_{3}$ : Roaming in the Dark or Roaming on the Ground? J. Phys. Chem. A 2015, 119, 7163-7168.

(44) Homayoon, Z.; Bowman, J. M.; Dey, A.; Abeysekera, C.; Fernando, R.; Suits, A. G., Experimental and Theoretical Studies of Roaming Dynamics in the Unimolecular Dissociation of $\mathrm{CH}_{3} \mathrm{NO}_{2}$ to $\mathrm{CH}_{3} \mathrm{O}+$ NO. Z. Phys. Chem. 2013, 227, 1267-1280.

(45) Dey, A.; Fernando, R.; Abeysekera, C.; Homayoon, Z.; Bowman, J. M.; Suits, A. G., Photodissociation Dynamics of Nitromethane and Methyl Nitrite by Infrared Multiphoton Dissociation Imaging with Quasiclassical Trajectory Calculations: Signatures of the Roaming Pathway. J. Chem. Phys. 2014, 140 (054305).

(46) Annesley, C. J.; Randazzo, J. B.; Klippenstein, S. J.; Harding, L. B.; Jasper, A. W.; Georgievskii, Y.; Ruscic, B.; Tranter, R. S., Thermal Dissociation and Roaming Isomerization of Nitromethane: Experiment and Theory. J. Phys. Chem. A 2015, 119, 7872-7893.

(47) Lu, Z.; Chang, Y. C.; Ying, Q.-Z.; Ng, C. Y.; Jackson, W. M., Evidence for Direct Molecular Oxygen Production in $\mathrm{CO}_{2}$ Photodissociation. Science 2014, 346, 61-64. 
(48) Nguyen, T.-N.; Putikam, R.; Lin, M. C., A Novel and Facile Decay path of Criegee Intermediates by Intramolecular Insertion Reactions via Roaming Transition States. $J$. Chem. Phys. 2015, 142, 124312.

(49) Joalland, B.; Shi, Y.; Kamasah, A.; Suits, A. G.; Mebel, A. M., Roaming Dynamics in Radical Addition-Elimination Reactions. Nature Comm. 2014, 5, 1-6.

(50) Pemerantz, A. E.; Camden, J. P., Chiou, A. S.,; Ausfelder, F.; Chawla, N.; Hase, W. L.; Zare, R. N., Reaction Products with Internal Energy beyond the Kinematic Limit Result from Trajectories Far from the Minimum Energy Path: An Example from $\mathrm{H}+$ $\mathrm{HBr}->\mathrm{H}_{2}+$ Br. J. Am. Chem. Soc. 2005, 127, 16368-16369.

(51) Coutinho, N. D.; Silva, V. H. C.; de Oliveira, H. C. B.; Camargo, A. J.; Mundim, K. C.; Aquilanti, V., Stereodynamical Origin of Anti-Arrhenius Kinetics: Negative Activation Energy and Roaming for a Four-Atom Reaction. J. Phys. Chem. Lett. 2015, 6, 15531558.

(52) Osborn, D. L., Exploring Multiple Reaction Paths to a Single Product Channel. Adv. Chem. Phys. 2008, 138, 213-265.

(53) Bowman, J. M.; Shepler, B. C., Roaming Radicals. Ann. Rev. Phys. Chem. 2011, 62, 531.

(54) Bowman, J. M., Roaming. Mol. Phys. 2014, 112, 2516-2528

(55) Conte, R.; Houston, P. L.; Bowman, J. M. Classical Trajectory Study of Energy Transfer in Collisions of Highly Excited Allyl Radical with Argon J. Phys. Chem. A 2013, 11\%, 14028-14041.

(56) Conte, R.; Houston, P. L.; Bowman, J. M. Communication: A Benchmark-Quality, Full-Dimensional Ab Initio Potential Energy Surface for Ar-HOCO J. Chem. Phys. 2014, 140, 151101. 
(57) Conte, R.; Houston, P. L.; Bowman, J. M. Trajectory Study of Energy Transfer and Unimolecular Dissociation of Highly Excited Allyl with Argon J. Phys. Chem. A 2014, $118,7742-7757$.

(58) Houston, P.L.; Conte, R.; Bowman, J. M. Collisional Energy Transfer in Highly Excited Molecules J. Phys. Chem. A , 2014, 118, 7758-7775.

(59) Qu, C.; Conte, R.; Houston, P.L.; Bowman, J. M. 'Plug and play' Full-Dimensional Ab Initio Potential Energy and Dipole Moment Surfaces and Anharmonic Vibrational Analysis for $\mathrm{CH}_{4}-\mathrm{H}_{2} \mathrm{O}$ Phys. Chem. Chem. Phys. 2015, 17, 8172-8181.

(60) Conte R.; Qu C.; Bowman J. M. Permutationally-Invariant Fitting of Many-body, Noncovalent Interactions with Application to Three-body Methane-Water-Water J. Chem. Theory Comput. 2015, 11, 1631-1638.

(61) Houston, P.L.; Conte, R.; Bowman, J. M. A Model For Energy Transfer in Collisions of Atoms with Highly Excited Molecules J. Phys. Chem. A 2015, 119, 4695-4710.

(62) Conte, R.; Houston, P. L.; Bowman, J. M. Trajectory and Model Studies of Collisions of Highly Excited Methane with Water Using an Ab Initio Potential J. Phys. Chem. A, 2015, 119, 12304-12317.

(63) Varandas, A. J. C., Trajectory Binning Scheme and Non-active Treatment of Zero-point Energy Leakage in Quasi-classical Dynamics. Chem. Phys. Lett. 2007, 459, 386-392.

(64) Feller, D.; Dupuis, M.; Garrett, B. C., Barrier for the $\mathrm{H}_{2} \mathrm{CO} \rightarrow \mathrm{H}_{2}+\mathrm{CO}$ Reaction: A Discrepancy between High-level Electronic Structure Calculations and Experiment. $J$. Chem. Phys. 2000, 113, 218-226.

(65) Chang, Y.-T.; Minichino, C.; Miller, W. H., Classical Trajectory Studies of the Molecular Dissociation Dynamics of Formaldehyde: $\mathrm{H}_{2} \mathrm{CO} \rightarrow \mathrm{H}_{2}+$ CO. J. Phys. Chem. 1992, 96, 4341-4356. 
(66) Tsai, P.-Y.; Lin, K. C., Insight into Photofragment Vector Correlation by a Multi-center Impulsive Model. Phys. Chem. Chem. Phys. 2015, 17, 19592-19601.

(67) Tsai, P.-Y.; Lin, K. C., Insight into the Photodissociation Dynamical Feature of Convential Transition State and Roaming Pathways by an Impulsive Model. J. Phys. Chem. A 2015, 119, 29-38.

(68) Klippenstein, S. J.; Georgievskii, Y.; Harding, L. B., Statistical Theory for the Kinetics and Dynamics of Roaming Reactions. J. Phys. Chem. A 2011, 115, 14370-14381.

(69) Andrews, D. U.; Kable, S. H.; Jordan, M. J. T., A Phase Space Theory for Roaming Reactions. J. Phys. Chem. A 2013, 117, 7631-7642.

(70) Ulusoy, I. S.; Stanton, J. F.; Hernandez, R., Effects of Roaming Trajectories on the Transition State Theory Rates of a Reduced-Dimensional Model of Ketene Isomerization. J. Phys. Chem. A 2013, 117, 7553-7560.

(71) Ulusoy, I. S.; Stanton, J. F.; Hernandez, R., Correction to "Effects of Roaming Trajectories on the Transition State Theory Rates of a Reduced-Dimensional Model of Ketene Isomerization. J. Phys. Chem. A 2013, 117, 10567-10568.

(72) Ulusoy, I. S.; Hernandez, R., Revisiting Roaming Trajectories in Ketene Isomerization at Higher Dimensionality. Theor. Chem. Acc. 2014, 133, 1528. 


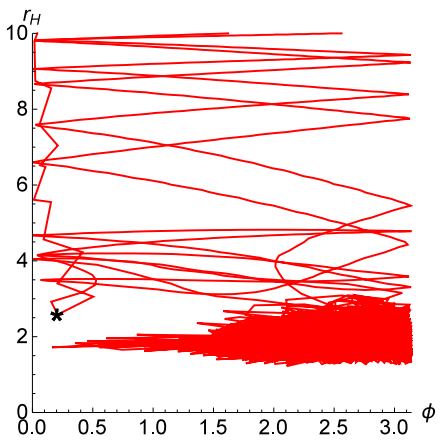

